\title{
Testing Efficient Risk Sharing with Heterogeneous Risk Preferences*
}

\author{
Maurizio Mazzocco \\ UCLA
}

\author{
Shiv Saini
}

Cornerstone Research

Current Draft, October 2010. First Draft, October 2005.

\begin{abstract}
Previous papers have tested efficient risk sharing under the assumption of identical risk preferences. In this paper we show that, if in the data households have heterogeneous risk preferences, the tests proposed in the past reject efficiency even if households share risk efficiently. To address this issue we propose a method that enables one to test efficiency even when households have different preferences for risk. The method is composed of three tests. The first one can be used to determine whether in the data under investigation households have homogeneous risk preferences. The second and third tests can be used to evaluate efficient risk sharing when the hypothesis of homogeneous risk preferences is rejected. We use this method to test efficient risk sharing in rural India. Using the first test, we strongly reject the hypothesis of identical risk preferences. We then test efficiency with and without the assumption of preference homogeneity. In the first case we reject efficient risk sharing at the village and caste level. In the second case we still reject efficiency at the village level, but we cannot reject this hypothesis at the caste level. This finding suggests that the relevant risk-sharing unit in rural India is the caste and not the village.
\end{abstract}

\section{Introduction}

A good understanding of the degree of risk sharing that characterizes households in industrialized and developing countries is important to answer policy questions. To see this consider for instance a village in a developing country. The consumption pattern and hence the welfare of each individual in the village is affected by a variety of idiosyncratic and aggregate shocks. Some examples of such shocks are severe weather conditions, price fluctuations, health problems, unemployment spells, and crop diseases. Unless the households in the village can insure themselves against these shocks using the existing institutions, individual consumption will fluctuate in response to them with detrimental

${ }^{*}$ We are grateful to Moshe Buchinsky, Pierre-André Chiappori, Flavio Cunha, Mariacristina De Nardi, Frederico Finan, James Heckman, Joseph Hotz, John Kennan, Dennis Kristensen, Rodolfo Manuelli, Robert Miller, Masao Ogaki, Jack Porter, Bernard Salanie, Sam Schulhofer-Wohl, Duncan Thomas, Jesus Fernandez-Villaverde, James Walker, and seminar participants at a number of venues for helpful comments. 
effects on individual welfare. The typical risk-sharing institutions available to households in rural villages are gifts and transfers, borrowing from village lenders, saving and storage technologies, and the diversification of crops. In addition to the existing institutions, the local and central government may decide to provide additional insurance for instance by introducing new financial assets, by simplifying the access to the existing financial markets, or by providing crop, health, and unemployment insurance.

A crucial step in evaluating whether there is scope for government reforms is the derivation of tests that enable one to determine whether households are able to share risk efficiently using the existing institutions. In the past two decades several papers have derived such tests and tested efficient risk sharing in industrialized and developing countries. The papers in this literature have one common feature. They assume that households have identical preferences for risk. In the present paper we show that, if in the data the preferences for risk are heterogeneous, the tests used in the past reject efficient risk sharing even if the households under investigation share risk efficiently.

Using this result as a starting point, this paper makes three main contributions. The first contribution is to provide two tests that enable one to evaluate efficient risk sharing even when households have heterogeneous preferences for risk. The tests that we propose are derived using a new approach. Instead of relying on the first order conditions, we use the household risk-sharing functions which relate household expenditure to aggregate resources. The use of the risk-sharing functions has four advantages. First, the heterogeneity in risk preferences can be easily considered in the derivation of the tests. Second, we are able to derive tests that are non-parametric. The only restrictions that the household utility functions must satisfy are monotonicity and concavity. Third, the non-separability between consumption and leisure can be easily incorporated in the tests. Lastly, the new approach enables us to construct a test that a researcher can use to determine whether the hypothesis of homogeneous risk preferences is rejected. If it is, one cannot use the old tests based on the assumption of identical preferences and new tests must be employed that allow for preference heterogeneity.

The second contribution of the paper is to show that the testable implications on which the two efficiency tests are based are the only implications of efficient risk sharing if the following two conditions are satisfied. First, the only assumptions on the household utility functions are monotonicity and concavity. Second, no longitudinal variation in wages is observed or used in the efficiency test. This result implies that any testable implication of efficient risk sharing different from the ones derived here must be the result of additional assumptions on household preferences or longitudinal variation in wages.

As a final contribution we use the method proposed in this paper to test efficient risk sharing in rural India. We find that it is crucial to allow for heterogeneity in risk preferences to understand the risk-sharing arrangements in Indian villages. Using data from the International Crops Research Institute for the Semi-Arid Tropics (ICRISAT) we strongly reject the hypothesis that households have identical preferences for risk. We then test efficient risk sharing at the village and caste level with and 
without the assumption of homogeneous risk preferences. At the village level, we reject efficiency in both cases. At the caste level, we reject the hypothesis that caste fellows share risk efficiently when we use the standard test. We cannot reject this hypothesis, however, when we use the non-parametric tests that allow for preference heterogeneity.

These findings suggest that the relevant risk-sharing unit in rural India is not the village, as previously suggested, but the caste. This result is consistent with recent evidence reported in Munshi and Rosenzweig (2009), where it is found that to understand migration patterns in rural India one should use the caste as the relevant social unit. In the last part of the paper we provide descriptive evidence on some of the institutions used by caste fellows to share risk. We find that transfers between households that belong to the same caste are important sources of mutual insurance in rural India.

The first paper to consider heterogeneity in risk preferences in testing full insurance is Altug and Miller (1990). In that paper the authors test efficiency by allowing the household preferences to depend on demographic variables. They therefore allow the curvature of the utility function to depend on observable characteristics. But conditional on the observables, the curvature cannot vary across households. Townsend (1994) partially addresses the issue of heterogeneity in risk preferences. His paper is divided into two parts. In one part, efficiency is tested under the assumption of identical risk preferences. This part is generally cited as evidence against efficient risk sharing. In the second part, the author allows for preference heterogeneity. This part has two limitations. First, it is assumed that preferences belong to the Constant Absolute Risk Aversion (CARA) class, which has been frequently criticized in the literature on decisions under uncertainty because of its limitations in describing household behavior. Second, when preference heterogeneity is allowed, the test is performed by estimating the efficiency condition using either six or ten observations. As a consequence the estimates on which the tests are based are imprecise and the outcome of the test difficult to interpret. Dubois (2004) also allows for heterogeneity in risk preferences when he analyzes share-cropping agreements. In the paper, households have Constant Relative Risk Aversion (CRRA) utilities and the heterogeneity in risk preferences is introduced by allowing the coefficient of relative risk aversion to depend on observable variables. The paper with more similarities to ours is the one by Schulhofer-Wohl (2010). Similarly to our paper, he proposes a test of efficient risk sharing that allows for heterogeneity in risk preferences. His analysis differs from ours in two respects. First, the test is parametric and only valid under a particular specification of the utility function. Second, he does not control directly for heterogeneity in risk preferences. Instead, the heterogeneity is captured by allowing for nuisance parameters in the model. The main advantage of Schulhofer-Wohl's method relative to ours is that it requires a shorter panel for its implementation. Mazzocco (2004) investigates the effect of heterogeneity in risk preferences on efficient risk sharing within a group. It is shown that with heterogeneous risk preferences efficient risk sharing can increase group savings even if it reduces the amount of uncertainty faced by the group. Hara, Huang, and Kuzmics (2006) analyze 
theoretically how the efficient risk-sharing rule is affected by the heterogeneity in risk attitude.

The paper proceeds as follows. In Section 2 we discuss the relationship between preference heterogeneity and the performance of the standard test of full insurance. In Section 3, we present a model of efficient risk sharing. In Section 4, we derive the testable implications of homogeneity in risk preferences and efficiency. In Section 5, we develop a method that can be used to measure the degree of risk sharing that characterizes a household if full insurance is rejected. In Section 6, we discuss the econometric issues related to the implementation of the tests. In Section 7, the data used in the tests are described. Section 8 reports and discusses the results of the tests. Section 9 concludes.

\section{Preference Heterogeneity and The Standard Efficiency Test}

In the past two decades many papers have tested efficient risk sharing using data from developed and developing countries. Some of the papers in this literature are Altug and Miller (1990), Cochrane (1991), Mace (1991), Altonji, Hayashi, and Kotlikoff (1992), Townsend (1994), Attanasio and Davis (1996), Hayashi, Altonji, and Kotlikoff (1996), Ravallion and Chaudhuri (1997), Ogaki and Zhang (2001), Amin, Rai, and Topa (2003), and Blundell, Pistaferri, and Preston (2006). The general interpretation of the findings in this literature is that efficient risk sharing is rejected. The large number of papers that develop models of partial insurance are evidence that this is the perception in the economic profession. ${ }^{1}$ The papers in the risk-sharing literature have one common feature. They assume that the households under investigation have the same risk preferences. In this section we will show that, if in the data households have heterogeneous preferences for risk, tests based on the assumption of identical risk preferences reject full insurance even if the households under consideration share risk efficiently.

To describe the effect of the assumption of identical risk preferences on previous tests, we will use the following simple example. Consider an economy composed of two households with heterogeneous preferences for risk which share risk efficiently. It is assumed that their preferences are separable across states of nature, over time, and between consumption and leisure. It is also assumed that they belong to the Harmonic Absolute Risk Aversion (HARA) class, i.e. the marginal utility of consumption can be written in the form $u_{c}^{i}(c)=\left(a_{i}+c\right)^{-\gamma_{i}}$. The economy is characterized by both idiosyncratic and aggregate shocks. To simplify the discussion, in this example we will assume that the only possible source of heterogeneity across households is represented by differences in the household utility functions.

The intuition behind the efficient allocation of resources in this economy can be provided by dividing risk sharing into two parts. First, if the households share risk efficiently it is optimal for them to pool their resources and hence eliminate the idiosyncratic uncertainty that they face. We will

\footnotetext{
${ }^{1}$ See for instance Kocherlakota (1996), Ligon (1998), Attanasio and Rios-Rull (2000), Ligon, Thomas, and Worrall (2002), and Heathcote, Storesletten, and Violante (2007).
} 
refer to this component of risk sharing as income pooling. Second, under efficiency the households should insure each other against aggregate shocks by allocating pooled resources according to their individual preferences for risk. This component of risk sharing will be denoted by the term mutual insurance.

Now suppose that the econometrician incorrectly assumes that the two households have identical risk preferences. We will show that in the economy under consideration mutual insurance has a significant effect on household behavior. The econometrician, however, by assuming that preferences are identical is imposing the restriction that there is no mutual insurance in the economy. We will show that this result holds by using two standard sets of conditions: the feasibility conditions and the efficiency conditions.

Denote by $t$ an arbitrary period, by $\omega_{t}$ a realization of a state of nature in that period, and by $h_{t}=\left(\omega_{1}, \ldots, \omega_{t}\right)$ a history of realizations. Let $c^{i}\left(h_{t}\right)$ be consumption of household $i, y^{i}\left(h_{t}\right)$ be the amount of resources available to household $i$, and define $Y\left(h_{t}\right)=y^{1}\left(h_{t}\right)+y^{2}\left(h_{t}\right)$. The feasibility condition for a history $h_{t}$ can then be written as follows:

$$
c^{1}\left(h_{t}\right)+c^{2}\left(h_{t}\right)=Y\left(h_{t}\right) .
$$

It indicates that under efficiency only pooled resources can affect household consumption. The efficiency condition for a history $h_{t}$ can be written in the following form:

$$
\mu_{1} u_{c}^{1}\left(c^{1}\left(h_{t}\right)\right)=\mu_{2} u_{c}^{2}\left(c^{2}\left(h_{t}\right)\right)
$$

where $\mu_{i}$ is the Pareto weight of household $i$. The efficiency condition shows that aggregate resources are optimally allocated according to individual preferences and Pareto weights.

Figures 1 and 2 use the feasibility and efficiency conditions to describe the efficient allocation of resources in the economy for different realizations of aggregate income $Y\left(h_{t}\right)$. To focus on the effect of preference heterogeneity, we will consider the case of identical Pareto weights. Similar results hold if the Pareto weights are allowed to differ. The dotted curve in Figure 1 depicts the weighted marginal utility of the more risk averse household, whereas the solid line describes the weighted marginal utility of the second household. Figure 1 can be used to determine the efficient allocation of resources for each realization of aggregate income using the following two steps. First, for a given $Y\left(h_{t}\right)$ draw a horizontal line to determine a pair of consumption levels at which the efficiency condition is satisfied. Second, move the horizontal line up or down until the two consumption levels satisfy the feasibility condition. Figure 2 is obtained by repeating this procedure for each realization of aggregate resources. It depicts efficient consumption for the two households as a function of aggregate resources. We will use the term risk-sharing functions to refer to the functions depicted in Figure 2.

The two figures show that if preferences are heterogenous the weighted marginal utilities will generally cross. As a consequence the risk-sharing functions will also cross. To provide some insight on the meaning of the crossing, consider the realizations of aggregate resources that generate household 
expenditures that are to the left of the crossing point. In this case the household that is more risk averse consumes more than half of aggregate resources. The allocations in this region can be interpreted as the outcome of the insurance provided by the less risk averse household against adverse realizations of aggregate resources. Consider now the region to the right of the crossing point. In this case, the household that is less risk averse consumes more than half of aggregate resources as a compensation for the insurance provided against adverse aggregate shocks. To summarize, in this economy the households first eliminate the idiosyncratic uncertainty by pooling their resources and then allocate them according to the individual preferences for risk to provide insurance against aggregate shocks.

We will now describe the efficient allocations of resources under the assumption made by the econometrician that households have identical risk preferences. Figures 3 and 4 depict efficient risk sharing for the general case of different Pareto weights. They indicate that the weighted marginal utilities and hence the household risk-sharing functions can never cross. The reason for this result is straightforward. Since it is assumed that the two households have identical risk preferences there is no scope for mutual insurance. As a consequence, the household with higher Pareto weight will always consume more than half of aggregate resources. We can therefore conclude that the assumption of identical preferences for risk is equivalent to the restriction that mutual insurance is an irrelevant component of efficient risk sharing.

Using this result we can determine how the tests developed in the risk-sharing literature perform when they are used to test efficiency in an economy characterized by heterogeneous risk preferences. To this end, consider the following generalization of the efficiency test initially proposed by Mace (1991). ${ }^{2}$ Under the assumptions made by the econometrician that the two households in the economy have identical HARA preferences and share risk efficiently, it is straightforward to show that the following relationship between household and aggregate consumption must be satisfied:

$$
f\left(c_{t+1}^{i}\right)-f\left(c_{t}^{i}\right)=\frac{1}{2} \sum_{j=1}^{2}\left(f\left(c_{t+1}^{j}\right)-f\left(c_{t}^{j}\right)\right),
$$

where $f(c)$ is a transformation of consumption that varies with the utility function chosen to characterize the household preferences. Specifically, the function $f$ takes the form $f(c)=c$ if one uses CARA preferences, $f(c)=\ln (c)$ under the assumption of CRRA preferences, and $f(c)=\ln (a+c)$ for the general class of HARA preferences. This generalization of Mace's test is useful because the tests used in the papers cited above are a special case of it. To see this note that under the assumption of CARA preferences, equation (1) establishes that the first difference in household consumption must equal the first difference in aggregate consumption, which is the test used in Mace (1991), Townsend (1994), and Ravallion and Chaudhuri (1997). If preferences are assumed to belong to the CRRA

\footnotetext{
${ }^{2}$ In her paper, Mace allows for preference shocks. For ease of exposition we abstract from them when we derive the general version of her test. The main conclusion of this section is not affected by this assumption.
} 
class, according to equation (1) household consumption growth must equal aggregate consumption growth, which is the test used in Cochrane (1991), Mace (1991), and Altonji, Hayashi, and Kotlikoff (1992). ${ }^{3}$ Finally if the utility function belongs to the general HARA class, equation (1) still equates household consumption growth with aggregate consumption growth, but the growth rate must be computed taking into account the subsistence level $a$. This test is used in Ogaki and Zhang (2001). ${ }^{4}$

Now consider two periods with the following features. In the first period the economy is characterized by an adverse realization of aggregate resources, i.e. aggregate resources are on the left of where the risk-sharing functions cross. In the second period the economy is characterized by a good realization of aggregate resources, i.e. aggregate resources are on the right of the crossing point. Since the two households have different preferences for risk, they will insure each other against aggregate shocks. This implies that between the two periods consumption of the more risk averse household will vary less than aggregate consumption and consumption of the less risk averse household will vary more. Formally, the equality tested in previous papers is replaced by the following inequalities:

$$
f_{1}\left(c_{t+1}^{1}\right)-f_{1}\left(c_{t}^{1}\right)<\frac{1}{2} \sum_{j=1}^{2}\left(f_{j}\left(c_{t+1}^{j}\right)-f_{j}\left(c_{t}^{j}\right)\right)<f_{2}\left(c_{t+1}^{2}\right)-f_{2}\left(c_{t}^{2}\right) .
$$

This implies that, if in the data used by previous papers households have heterogeneous risk preferences, efficient risk sharing should have been rejected even if households share risk efficiently. ${ }^{5}$

One additional aspect of the tests used in the past should be discussed before claiming that heterogeneity in risk preferences may explain previous rejections. In the risk-sharing literature the efficiency condition (1) is tested by adding a variable that captures idiosyncratic shocks to the equation and by verifying whether the coefficient on this variable is statistically significant. Most of the papers add changes in household income and they find that the coefficient is statistically significant and positive. It is important to understand whether heterogeneity in risk preferences can explain the positive coefficient.

The next proposition considers the class of HARA preferences employed in previous papers and it shows that heterogeneity in the curvature parameter $\gamma$ can explain the positive coefficient on changes in income if less risk averse households choose income processes that vary more with aggregate shocks. ${ }^{6}$

\footnotetext{
${ }^{3}$ Altug and Miller (1990), Hayashi, Altonji, and Kotlikoff (1996), and in part of their work Attanasio and Davis (1996) use preferences that are non-separable between consumption and leisure. The intuition provided in this section applies also to those papers. However, a model with nonseparable preferences allows for more general patterns of household consumption. We consider this more general case starting from the next section.

${ }^{4}$ Ogaki and Zhang (2001) apply the test to two different sets of data: the data collected by the International Food Policy Research Institute (IFPRI) for Pakistani households and the ICRISAT data for Indian households. When they use the test initially introduced by Mace (1991) they do not reject efficiency in Pakistani villages, but they reject this hypothesis for two of the Indian villages. These findings are consistent with the discussion of this section. Homogeneity in risk preferences can be a good assumption in some environments, but a bad assumption in others.

${ }^{5}$ The test proposed by Cochrane (1991) is affected by the same problem. The assumption of identical CRRA preferences enables one to include in the constant the terms that capture aggregate quantities, $\left(1 / \gamma_{j}\right) \ln \left(\mu_{t+1} / \mu_{t}\right)$ in equation (8) in Cochrane (1991). If preferences are heterogeneous the constant will generally be smaller for more risk averse households and larger for less risk averse households. Therefore the inequality will generally still hold. It is important to point out that Cochrane (1991) recognizes that in the presence of preference heterogeneity standard risk sharing regressions suffer from an omitted variable bias.

${ }^{6}$ Schulhofer-Wohl (2010) discusses a similar result for CRRA preferences. He also provides evidence that more risk
} 
Proposition 1 Consider an economy in which (i) households share risk efficiently, (ii) they are characterized by preferences that belong to the HARA class, (iii) there is heterogeneity in the curvature parameter $\gamma$, (iv) conditional on a realization of the aggregate shocks, the expected value of $\Delta y^{i}$ computed with respect to the idiosyncratic shocks is decreasing in $\gamma_{i}$ if $\Delta Y_{t} \geq 0$ and increasing in $\gamma_{i}$ if $\Delta Y_{t}<0$. Then in a least squared regression of the following equation:

$$
\Delta f\left(c_{t}^{i}\right)-\frac{1}{n} \sum_{j=1}^{n} \Delta f\left(c_{t}^{j}\right)=\xi \Delta y_{t}^{i}+\epsilon_{t},
$$

the expected value of the estimated coefficient $\xi$ is positive.

Proof. In the appendix.

The intuition behind the result of Proposition 1 is straightforward. If the economy is characterized by households with heterogeneous curvature parameters, there is an omitted variable from equation (2). It measures the variation in household consumption which is not captured by the variation in aggregate resources. Denote with $e$ this variable. Standard results on omitted variable biases indicate that

$$
E(\hat{\xi})=\xi+\operatorname{VAR}\left(\Delta y_{t}^{i}\right)^{-1} \operatorname{Cov}\left(\Delta y_{t}^{i}, e_{t}^{i}\right)
$$

In an economy in which households share risk efficiently, $\xi$ equals zero. Consequently the expected value of $\hat{\xi}$ is positive if the covariance between $\Delta y_{t}^{i}$ and the omitted variable is positive. To see that this is the case, consider first an economy in which the aggregate shock in period $t+1$ is worse than the one realized in period $t$, which implies $\Delta Y_{t}<0$. Observe that households with low $\gamma$ provide insurance against adverse aggregate shocks. Hence, in this economy their consumption decreases more than aggregate consumption and their $e$ is negative. Using the same argument, $e$ must be positive for households with high $\gamma$. Under the assumption that less risk averse households have income processes that are affected more by reductions in aggregate resources, $\Delta y_{t}^{i}$ is negative and smaller for households with low $\gamma$. As a consequence, conditional on $\Delta Y_{t}<0$, the covariance between $e$ and $\Delta y_{t}^{i}$ is positive. Consider now an economy in which $\Delta Y_{t} \geq 0$. In this case, since the consumption of households with low $\gamma$ varies more than aggregate resources, $e_{t}^{i}$ is positive for them and negative for households with high $\gamma$. Moreover, $\Delta y_{t}^{i}$ is positive and larger for households with low $\gamma$. As a result, conditional on $\Delta Y_{t}>0$, the covariance between $e_{t}^{i}$ and $\Delta y_{t}^{i}$ is positive. All this implies that the unconditional covariance between $e_{t}^{i}$ and $\Delta y_{t}^{i}$ is positive and the expected value of the coefficient on changes in income is positive. ${ }^{7}$

averse individuals have riskier income processes using the PSID. Similar evidence is provided in Fuchs-Schundeln and Schundeln (2005) using German data.

${ }^{7}$ If the economy is charaterized by heterogeneity in the discount factors, a result similar to the one presented in Proposition 1 holds. With heterogeneous discount factors, equation (2) is characterized by an omitted variable that is a function of the difference between the discount factor of household $i$ and the average discount factor in the economy. If this omitted variable is correlated with household income, the standard test will reject full insurance even if it is satisfied. Moreover, the sign on the coefficient on changes in household income will depend on the correlation between the omitted variable and the changes in income. 
Finally, observe that in an economy with full insurance one should expect households that are less risk averse to choose income processes that vary more with aggregate shocks if households select their income process before entering the risk-sharing agreement and the reallocation of the income processes is costly. ${ }^{8}$

The discussion in this section indicates that if in the economy under investigation preferences for risk are heterogeneous the tests used in the past reject full insurance even if households share risk efficiently. It is therefore important to derive a test that enables one to verify whether the null of homogeneity in risk preferences is rejected in the data. If it is, a test of efficiency is required that allows for differences in risk preferences. The rest of the paper is devoted to deriving tests that enable one to evaluate the hypotheses of homogeneity in risk preferences and efficiency.

\section{$3 \quad$ A Model of Efficient Risk Sharing}

We use a standard model to characterize efficient risk sharing. In this section we outline its main features and we derive a new result which is crucial for setting up the homogeneity and efficiency tests. Consider an economy in which households live for $\tau$ periods. For a given history of realizations $h_{t}$, let $w_{t}^{i}\left(h_{t}\right)$ be the labor productivity of household $i$ in period $t$ and denote with $T_{t}^{i}\left(h_{t}\right)$ the total amount of time that can be divided between leisure and labor. The aggregate amount of non-labor resources in the economy is denoted by $Y_{t}\left(h_{t}\right)$, where $Y_{t}\left(h_{t}\right)$ may include profits and savings. Let $c_{t}^{i}\left(h_{t}\right)$ and $l_{t}^{i}\left(h_{t}\right)$ be, respectively, consumption and leisure of household $i$ in period $t$ conditional on the history $h_{t}$. Household preferences are assumed to be separable over time and across states of nature. They are allowed to depend on observable and unobservable heterogeneity, which will be denoted by $z_{t}^{i}\left(h_{t}\right)$ and $\eta_{t}^{i}\left(h_{t}\right)$. The corresponding utility function $u^{i}\left[c_{t}^{i}\left(h_{t}\right), l_{t}^{i}\left(h_{t}\right) ; z_{t}^{i}\left(h_{t}\right), \eta_{t}^{i}\left(h_{t}\right)\right]$ is assumed to be strictly increasing, strictly concave, and twice continuously differentiable in consumption and leisure. Households have a common discount factor $\beta$ and share the same beliefs over histories of realizations, which are denoted by $P\left(h_{t}\right)$.

Efficient risk sharing in this economy can be described using a standard Pareto problem. Let $\mu_{i}$ be the Pareto weight assigned to household $i$ with $\sum_{i}^{n} \mu_{i}=1$ and suppose for simplicity that $0<\mu_{i}<1$. The efficient allocation of resources is then the solution of the following problem:

$$
\begin{gathered}
\max _{\left\{c_{t}^{i}\left(h_{t}\right), l_{t}^{i}\left(h_{t}\right)\right\}} \sum_{i=1}^{n} \mu_{i} \sum_{t=1}^{\tau} \beta^{t} \sum_{h_{t}} P\left(h_{t}\right) u^{i}\left[c_{t}^{i}\left(h_{t}\right), l_{t}^{i}\left(h_{t}\right) ; z_{t}^{i}\left(h_{t}\right), \eta_{t}^{i}\left(h_{t}\right)\right] \\
\text { s.t. } \sum_{i=1}^{n}\left(c_{t}^{i}\left(h_{t}\right)+w_{t}^{i}\left(h_{t}\right) l_{t}^{i}\left(h_{t}\right)\right)=Y_{t}\left(h_{t}\right)+\sum_{i=1}^{n} w_{t}^{i}\left(h_{t}\right) T_{t}^{i}\left(h_{t}\right) \quad \text { for each } t, h_{t} \\
c_{t}^{i}\left(h_{t}\right)>0, \quad 0 \leq l_{t}^{i}\left(h_{t}\right) \leq T_{t}^{i}\left(h_{t}\right) \quad \text { for each } t, h_{t},
\end{gathered}
$$

\footnotetext{
${ }^{8}$ Observe that efficiency is not violated if the reallocation of the income processes or occupations is costly since transfers can undo all the effects that a particular allocation has on household welfare.
} 
where the right hand side of the resource constraint is full income in the economy.

The test of homogeneous risk preferences and the two efficiency tests will be derived using the risk-sharing functions discussed in the previous section and depicted in Figures 2 and 4. With nonseparability between consumption and leisure, household expenditure in period $t$ is equal to $c_{t}^{i}+w_{t}^{i} l_{t}^{i}$. Therefore, one possible approach that can be used to derive the risk-sharing functions is to solve the Pareto problem (3) and obtain the consumption and leisure functions. These functions can then be substituted in $c_{t}^{i}+w_{t}^{i} l_{t}^{i}$ to recover the risk-sharing functions. This approach has one major limitation. The risk-sharing functions would depend on the labor productivities and heterogeneity variables of each household in the economy, i.e.

$$
\rho_{t}^{i}=c_{t}^{i}+w_{t}^{i} l_{t}^{i}=\bar{\rho}^{i}\left(\bar{Y}_{t} ; w_{t}^{1}, \ldots, w_{t}^{n}, z_{t}^{1}, \ldots, z_{t}^{n}, \eta_{t}^{1}, \ldots, \eta_{t}^{n}\right) \quad \text { for } i=1, \ldots, n
$$

where $\bar{Y}$ is full income. ${ }^{9}$ A test based on these risk-sharing functions is not feasible for two reasons. First, in every dataset one only observes a fraction of the households that compose the economy. Some of the variables in the risk-sharing functions are therefore not observed. Second, even if all the variables were observed it would generally be infeasible to estimate a function that depends on so many variables. We solve this problem by using a three-stage formulation of the economy which has the same solution as the standard Pareto program.

We will now describe the three-stage formulation starting from the last stage. Let $\rho_{t}^{i}\left(h_{t}\right)$ be an arbitrary amount of aggregate resources allocated by the social planner to household $i$ in period $t$ conditional on the history $h_{t}$. In the last stage, conditional on $\rho_{t}^{i}\left(h_{t}\right)$ household $i$ chooses consumption and leisure for period $t$ and history $h_{t}$ by solving the following individual problem:

$$
\begin{gathered}
V^{i}\left(\rho_{t}^{i}\left(h_{t}\right) ; w_{t}^{i}\left(h_{t}\right), z_{t}^{i}\left(h_{t}\right), \eta_{t}^{i}\left(h_{t}\right)\right)=\max _{c_{t}^{i}\left(h_{t}\right), l_{t}^{i}\left(h_{t}\right)} u^{i}\left[c_{t}^{i}\left(h_{t}\right), l_{t}^{i}\left(h_{t}\right) ; z_{t}^{i}\left(h_{t}\right), \eta_{t}^{i}\left(h_{t}\right)\right] \\
\text { s.t. } c_{t}^{i}\left(h_{t}\right)+w_{t}^{i}\left(h_{t}\right) l_{t}^{i}\left(h_{t}\right)=\rho_{t}^{i}\left(h_{t}\right) \\
c_{t}^{i}\left(h_{t}\right) \geq 0, \quad 0 \leq l_{t}^{i}\left(h_{t}\right) \leq T_{t}^{i}\left(h_{t}\right) .
\end{gathered}
$$

Consider now the second stage. Let $\rho_{t}^{i, j}\left(h_{t}\right)$ denote an arbitrary amount of aggregate resources allocated by the planner to the pair composed of households $i$ and $j$. In the intermediate stage, conditional on $\rho_{t}^{i, j}\left(h_{t}\right)$ the pair chooses the optimal amount of resources to allocate to households $i$ and $j$ in period $t$ and history $h_{t}$ by solving the following problem: ${ }^{10}$

$$
\begin{gathered}
V^{i, j}\left(\rho_{t}^{i, j} ; w_{t}^{i}, w_{t}^{j}, z_{t}^{i}, z_{t}^{j}, \eta_{t}^{i}, \eta_{t}^{j}\right)=\max _{\rho_{t}^{i}, \rho_{t}^{j}} \mu_{i} V^{i}\left(\rho_{t}^{i} ; w_{t}^{i}, z_{t}^{i}, \eta_{t}^{i}\right)+\mu_{j} V^{j}\left(\rho_{t}^{j} ; w_{t}^{j}, z_{t}^{j}, \eta_{t}^{j}\right) \\
\text { s.t. } \rho_{t}^{i}+\rho_{t}^{j}=\rho_{t}^{i, j} .
\end{gathered}
$$

\footnotetext{
${ }^{9}$ Note that the assumption that preferences are separable over time and across states enables us to write the risksharing functions only as a function of variables in period $t$ and history $h_{t}$.

${ }^{10}$ Unless required for expositional clarity, the dependence on $h_{t}$ will be suppressed in the rest of the paper.
} 
It is worth discussing two features of the second-stage problem. First, since $\rho_{t}^{k}=c_{t}^{k}+w_{t}^{k} l_{t}^{k}$, its solution provides the risk-sharing functions on which the tests will be based. Second, the risk-sharing functions obtained using this stage are only function of the labor productivities and heterogeneity variables of households $i$ and $j$, i.e.

$$
\rho_{t}^{k}=\rho_{t}^{k}\left(\rho_{t}^{i, j}, w_{t}^{i}, w_{t}^{j} ; z_{t}^{i}, z_{t}^{j}, \eta_{t}^{i}, \eta_{t}^{j}\right) \quad \text { for } k=i, j
$$

Since in many datasets one observes wages and heterogeneity variables for all pairs of households in the sample, tests based on the risk-sharing functions are feasible as long as one uses the risk-sharing functions obtained in the intermediate stage.

In the first stage, the social planner allocates optimally full income to each pair of households. Conditional on the amount of resources available in the economy in period $t$ and history $h_{t}$, each pair receives an allocation that is the solution of the following problem: ${ }^{11}$

$$
\begin{gathered}
V\left(Y_{t}+\sum_{i=1}^{n} w_{t}^{i} T_{t}^{i} ; w_{t}, z_{t}, \eta_{t}\right)=\max _{\left\{\rho_{t}^{2 i-1,2 i}\right\}} \sum_{i=1}^{n / 2} V^{2 i-1,2 i}\left(\rho_{t}^{2 i-1,2 i} ; w_{t}^{2 i-1}, w_{t}^{2 i}, z_{t}^{2 i-1}, z_{t}^{2 i}, \eta_{t}^{2 i-1}, \eta_{t}^{2 i}\right) \\
\text { s.t. } \sum_{i=1}^{n / 2} \rho_{t}^{2 i-1,2 i}=Y_{t}+\sum_{i=1}^{n} w_{t}^{i} T_{t}^{i},
\end{gathered}
$$

where $w_{t}, z_{t}$, and $\eta_{t}$ are the vectors of labor productivities and heterogeneity variables.

Under the standard assumptions that preferences are separable over time and across states of nature, the solution of the three-stage problem is also the solution of the original Pareto problem. The next proposition states the result.

Proposition 2 The solution of the three-stage formulation is the solution of the Pareto problem (3).

Proof. In the appendix.

We conclude the section by providing the intuition for why the risk-sharing functions obtained using the three-stage formulation have a simpler functional form. Observe that $\rho^{i j}$ is the solution of the first stage problem. As a consequence it depends on all the exogenous variables that characterize the economy. This implies that the risk-sharing functions obtained with the three-stage formulation depend on the same number of variables that characterize the ones obtained using the Pareto problem. But when the three-stage formulation is used, the risk-sharing functions of households $i$ and $j$ are affected by the vector of labor productivities and heterogeneity variables of the other households only through $\rho^{i j}$.

\footnotetext{
${ }^{11}$ The Pareto problem can be decomposed in three stages by pairing households in different ways. Here we consider one possible set of pairs under the assumption that there is an even number of households in the economy. If $n$ is odd, three households will have to be arranged in one group.
} 


\section{Testable Implications}

In this section we derive the testable implications on which the test of homogeneity in risk preferences and the efficiency tests are based. They are all derived using the risk-sharing functions obtained from the intermediate stage of the efficiency problem described in the previous section. The discussion will be divided into three parts. In the first part we will consider an economy where households share risk efficiently and derive a restriction that will enable us to test whether preferences are homogeneous across households. In the second part we will formulate a necessary condition of efficient risk sharing that can be used to test efficiency even if preferences are heterogeneous. In the last part, we will show that the necessary condition is also sufficient if no intertemporal variation in labor productivities is observed or used.

Before discussing in details the testable implications, we will provide the main idea on which they are based. Consider the pair composed of households $i$ and $j$. Preference homogeneity and efficiency are tested in two steps. We first recover the expenditure of household $i$ and, separately, of household $j$ as a function of the aggregate resources for the pair. We then test whether homogeneity in preferences and efficiency are rejected for this pair by considering two features of the risk-sharing functions: whether they cross and whether they are monotonically increasing. As argued in Section 2 , one or more crossings is suggestive of a rejection of the hypothesis of homogeneous preferences. We will show that a violation of monotonicity is indicative of a rejection of the hypothesis of efficient risk sharing. Figure 5 illustrates how these two features can be used to detect rejections of homogeneity and efficiency for the households that we observe in the data. They depict the estimated risk-sharing functions for three pairs of households in the ICRISAT. In the first panel we consider two households whose estimated risk-sharing functions do not cross and are monotonically increasing. For this pair we will not be able to reject preference homogeneity and efficiency. The second panel considers a pair of households with monotonically increasing risk-sharing functions that cross. For these two households, we cannot rule out the hypothesis of efficiency, but there is suggestive evidence that the two households have heterogeneous preferences. ${ }^{12}$ In the third panel, we report the risk-sharing functions for a last pair of households. In this case the risk-sharing function of the first household is decreasing for some values of aggregate resources. This pattern provides suggestive evidence that the households do not share risk efficiently. The rest of the section formalizes the idea illustrated in this paragraph.

We will first derive the testable implication for homogeneity in risk preferences. Consider households $i$ and $j$ and suppose that they share risk efficiently. As discussed in the first part of the paper, if their risk-sharing functions cross, mutual insurance must be a significant component of efficient risk sharing. If mutual insurance is an important feature of risk sharing, the two households must have

\footnotetext{
${ }^{12}$ The evidence is only suggestive because the risk-sharing functions have been estimated and the crossing may not be statistically significant.
} 
heterogeneous preferences. One can therefore conclude that under efficiency if the risk-sharing functions cross the hypothesis of homogeneous risk preferences can be rejected. This idea is formalized in the following proposition.

Proposition 3 Suppose that households $i$ and $j$ share risk efficiently. If there exist two realizations of aggregate resources $\rho^{i, j}$ and $\bar{\rho}^{i, j}$ such that

$$
\rho^{i}\left(\rho^{i, j}, w^{i}, w^{j} ; z^{i}, z^{j}, \eta^{i}, \eta^{j}\right)>\rho^{j}\left(\rho^{i, j}, w^{i}, w^{j} ; z^{i}, z^{j}, \eta^{i}, \eta^{j}\right)
$$

and

$$
\rho^{i}\left(\bar{\rho}^{i, j}, w^{i}, w^{j} ; z^{i}, z^{j}, \eta^{i}, \eta^{j}\right)<\rho^{j}\left(\bar{\rho}^{i, j}, w^{i}, w^{j} ; z^{i}, z^{j}, \eta^{i}, \eta^{j}\right)
$$

household $i$ and household $j$ cannot have identical preferences.

Proof. In the appendix.

Two remarks are in order. First, the preference homogeneity test is valid only under the maintained assumption that households share risk efficiently. The test is therefore useful only if the final goal is to test efficiency. Second, if homogeneity in risk preferences is rejected, efficient risk sharing cannot be tested using the standard approach and new tests of full insurance are required that allow for this type of heterogeneity.

We will now derive a necessary condition of efficient risk sharing that is satisfied even if households have different risk preferences. Consider households $i$ and $j$ and observe that under efficiency the following two restrictions must be fulfilled. First, after controlling for differences across households in labor productivities, observable and unobservable heterogeneity, only aggregate resources should affect the expenditure of households $i$ and $j$. Hence, conditional on $w^{i}, w^{j}, z^{i}, z^{j}, \eta^{i}$, and $\eta^{j}$, for each realization of $\rho^{i, j}$ only one value should be observed for $\rho^{i}\left(\rho^{i, j}, w^{i}, w^{j} ; z^{i}, z^{j}, \eta^{i}, \eta^{j}\right)$ and $\rho^{j}\left(\rho^{i, j}, w^{i}, w^{j} ; z^{i}, z^{j}, \eta^{i}, \eta^{j}\right)$. Second, an increase in aggregate resources should increase expenditure of both households. If one of these restrictions is not satisfied, household behavior is not only affected by changes in aggregate resources as predicted by efficient risk sharing, but also by idiosyncratic shocks. These two restrictions imply that a necessary condition for efficient risk sharing is that $\rho^{i}\left(\rho^{i, j}, w^{i}, w^{j} ; z^{i}, z^{j}, \eta^{i}, \eta^{j}\right)$ and $\rho^{j}\left(\rho^{i, j}, w^{i}, w^{j} ; z^{i}, z^{j}, \eta^{i}, \eta^{j}\right)$ must be strictly increasing functions of aggregate resources. The following proposition formalizes this result.

Proposition 4 Suppose that the utility functions of households $i$ and $j$ are strictly increasing and concave. Then, if households $i$ and $j$ share risk efficiently, $\rho^{i}\left(\rho^{i, j}, w^{i}, w^{j} ; z^{i}, z^{j}, \eta^{i}, \eta^{j}\right)$ and $\rho^{j}\left(\rho^{i, j}, w^{j}, w^{i} ; z^{i}, z^{j}, \eta^{i}, \eta^{j}\right)$ are strictly increasing functions of aggregate resources.

Proof. In the appendix.

The result presented in Proposition 4 contains two testable implications. First, after controlling for aggregate resources, labor productivities, observable and unobservable heterogeneity, the risk-sharing 
functions should not depend on other variables that capture idiosyncratic shocks. This testable implication is a generalization of the standard test of efficiency to an environment with heterogeneous risk preferences. The second testable implication can be described as follows. After controlling for labor productivities, observable and unobservable heterogeneity, the risk-sharing functions should be increasing in aggregated resources. To the best of our knowledge this is the first paper to propose this restriction as a test of efficiency. It has one advantage relative to the first implication. It does not require the choice of a variable that captures idiosyncratic shocks. This choice is problematic for two reasons. First, it is often arbitrary. Second, some of the variables employed in the past to capture idiosyncratic shocks are endogenous. As argued in Ham and Jacobs (2000), the endogeneity of these variables can generate false rejections of efficiency. A test based on the second implication is not affected by these problems.

The condition described in Proposition 4 is also sufficient for efficient risk sharing if no intertemporal variation in labor productivities is observed or used to test efficiency. There are two situations in which this assumption on labor productivities is satisfied. First, no longitudinal variation in labor productivities is observed by the researcher or the researcher observes longitudinal variation in labor productivities but this variation is not used in the test. Second, household preferences are assumed to be separable between consumption and leisure, which implies that the variation in labor productivities is not exploited in the efficiency tests. These two situations are the ones considered by the risk-sharing literature. Mace (1991), Townsend (1994), Altonji, Hayashi, and Kotlikoff (1992), Ravallion and Chaudhuri (1997), and Ogaki and Zhang (2001) assume separability between consumption and leisure. Cochrane (1991) uses a cross-section of households and hence ignores any longitudinal variation in labor productivities. Altug and Miller (1990) and Hayashi, Altonji, and Kotlikoff (1996) exploit longitudinal consumption variation after controlling for longitudinal variation in leisure. This is equivalent to using longitudinal variation in consumption after having removed the portion that is explained by longitudinal variation in labor productivities. The next proposition formally describes the sufficient condition for efficiency.

Proposition 5 Suppose that preferences are separable between consumption and leisure or $w^{i}$ and $w^{j}$ do not vary. Then, if $\rho^{i}\left(\rho^{i, j}, w^{i}, w^{j} ; z^{i}, z^{j}, \eta^{i}, \eta^{j}\right)$ and $\rho^{j}\left(\rho^{i, j}, w^{j}, w^{i} ; z^{i}, z^{j}, \eta^{i}, \eta^{j}\right)$ are strictly increasing functions of aggregate resources, there exist utility functions that are strictly increasing and concave and Pareto weights such that the two households share risk efficiently.

Proof. In the appendix.

Proposition 5 implies that if only variation in expenditure is used, the only testable implications of efficient risk sharing are the ones described in Proposition 4. Any other restriction is the outcome of the particular functional form selected for household preferences. ${ }^{13}$

\footnotetext{
${ }^{13}$ Hara (2006) derives a similar result under the assumption that preferences are separable between consumption and
} 
Before concluding this section we will discuss two issues related to the assumption of identical discount factors. First, if the households have identical preferences but different discount factors the risk-sharing functions cannot cross. This result is presented in the following proposition.

Proposition 6 Suppose that two households have identical preferences but heterogenous discount factors. In addition, assume that they share risk efficiently. Then their risk-sharing functions will never cross.

Proof. In the appendix.

This result implies that, under the null of efficient risk sharing, if one rejects the hypothesis that the risk-sharing functions do not cross, households must have heterogeneous preferences. The intuition behind this result is straightforward. In each period $t$ the heterogeneous discount factors affect the efficient allocation of resources only through the ratio of the discount factors at the power of $t$. The effect is therefore similar to the one generated by different Pareto weights. The household with the higher discount factor receives a larger fraction of resources independently of the realization of aggregate resources. As a consequence, the risk-sharing functions can never cross. The only difference with respect to the Pareto weights is that the effect of the heterogeneity in discount factors changes with time. Therefore, the effect persists even after computing the first difference of household consumption.

As a second remark, observe that theoretically the method developed in this paper can easily allow for heterogeneity in the discount factors. One has simply to add the variable time, $t$, to the risk-sharing functions and the arguments made in the remaining sections do not change. Empirically, however, it is more difficult to implement the tests if time is added to these functions. For this reason, this generalization is left for future research.

We conclude this section by pointing out that in the case of no unobservable heterogeneity, the condition derived in Proposition 3 can be used to test the null of homogeneous risk preferences by simply looking at the data. No estimation is required. A similar argument holds for the monotonicity condition derived in Proposition 4. Unobservable heterogeneity, however, is known to affect any standard set of data. Starting from Section 6, we will discuss how this type of heterogeneity can be taken into consideration in the implementation of the tests.

\section{Computing the Degree of Risk Sharing If Efficiency Is Rejected}

If efficiency is rejected, one may want to determine the extent of risk sharing for the group of households under consideration. In this section, we outline a non-parametric method that enables one to

leisure. Our result is more general in the following sense. We show that without separability between consumption and leisure the condition that expenditure is an increasing function of aggregate resources is sufficient for efficient risk sharing only if no intertemporal variation in labor productivities is used. 
measure it. We will first discuss the case of no measurement errors and then we will introduce them in consumption and income. ${ }^{14}$

Full income of household $i$ can be written as a function of the aggregate shock $A_{t}$ and of the idiosyncratic shock $\epsilon_{t}^{i}$, i.e.

$$
y f_{t}^{i}=y_{t}^{i}+w_{t}^{i} T_{t}^{i}=f\left(A_{t}, \epsilon_{t}^{i}\right) .
$$

Two frequently used specifications for the function $f$ are $f\left(A_{t}, \epsilon_{t}^{i}\right)=q_{1}\left(A_{t}\right)+q_{2}\left(\epsilon_{t}\right)$ and $f\left(A_{t}, \epsilon_{t}^{i}\right)=$ $q_{1}\left(A_{t}\right) q_{2}\left(\epsilon_{t}\right) .{ }^{15}$ Without the assumption of efficient risk sharing, household $i$ 's expenditure depends on the aggregate as well as the idiosyncratic shocks, i.e.

$$
\rho_{t}^{i}=g^{i}\left(A_{t}, \epsilon_{t}^{i}, w_{t}^{i}, W_{t}^{-i}\right)
$$

where $W_{t}^{-i}$ is the vector of household wages in the group with the exclusion of $w_{t}^{i}$.

We will now consider two extreme cases. The first one corresponds to a situation in which a household is in autarky and there is no saving technology. In this case, household expenditure equals household full income and the degree of risk sharing is zero. The second case corresponds to a situation in which households share risk efficiently. In this case, the expenditure of each household is independent of the idiosyncratic shocks and the group is characterized by the highest degree of risk sharing. Using these two cases we can construct a measure of the degree of insurance.

In autarky $\rho_{t}^{i}=y f_{t}^{i}=f\left(A_{t}, \epsilon_{t}^{i}\right)$. Hence, the variance of household expenditure conditional on the aggregate shock and the wages captures the variation in income that is generated by the idiosyncratic shocks, i.e.

$$
\operatorname{Var}\left(\rho_{t}^{i} \mid A_{t}, w_{t}^{i}, W_{t}\right)=\operatorname{Var}\left(y f_{t}^{i} \mid A_{t}, w_{t}^{i}, W_{t}\right)=\operatorname{Var}\left(f\left(A_{t}, \epsilon_{t}^{i}\right) \mid A_{t}, w_{t}^{i}, W_{t}\right)
$$

This variance can be interpreted as the maximum amount of idiosyncratic risk that can be born by household $i$. Under full insurance, the same conditional variance is equal to zero since the household expenditure is independent of the idiosyncratic shocks. This result is consistent with the previous interpretation of the conditional variance. It measures the amount of idiosyncratic risk that is born by the household, which is zero in the full insurance case.

A natural measure of the degree of risk sharing can therefore be constructed in the following way. One can first determine the difference between the conditional variance of full income, which captures the idiosyncratic risk borne by the household, and the conditional variance of expenditure, which captures the residual idiosyncratic risk. This quantity provides a measure of the amount of idiosyncratic risk the household is able to insure against. One can then divide the difference by the conditional variance of full income so that the result is normalized to be between 0 and 1 . The degree

\footnotetext{
${ }^{14}$ For expositional purposes in this section we will suppress the dependence on the observable and unobservable heterogeneity.

${ }^{15}$ For examples of the additive specification see Aiyagari and Gertler (1991) and Lucas (1994). For a discussion of the multiplicative specification see for instance Krueger and Lustig (2010).
} 
of risk sharing can therefore be determined by computing the following quantity:

$$
\xi_{4}=\frac{\operatorname{Var}\left(y f_{t}^{i} \mid A_{t}, w_{t}^{i}, W_{t}\right)-\operatorname{Var}\left(\rho_{t}^{i} \mid A_{t}, w_{t}^{i}, W_{t}\right)}{\operatorname{Var}\left(y f_{t}^{i} \mid A_{t}, w_{t}^{i}, W_{t}\right)} .
$$

If the households share risk efficiently, $\xi_{4}$ will be equal to 1 . If the household is in autarky, $\xi_{4}$ will have a value of 0 . For every other case $\xi_{4}$ will be between these two extreme values.

Suppose now that the expenditure data are affected by an additive measurement error $m^{i}$. In this case the variance of expenditure is the sum of the variance of true expenditure and of the variance of the measurement error, i.e.

$$
\operatorname{Var}\left(\rho_{t}^{i} \mid A_{t}, w_{t}^{i}, W_{t}\right)=\operatorname{Var}\left(\rho_{t}^{T, i} \mid A_{t}, w_{t}^{i}, W_{t}\right)+\operatorname{Var}\left(m_{t}^{i} \mid A_{t}, w_{t}^{i}, W_{t}\right)
$$

As a consequence, the method discussed above cannot be applied directly. Observe, however, that for households that satisfy the full insurance hypothesis the conditional variance of true expenditure is equal to 0 . This implies that,

$$
\operatorname{Var}\left(\rho_{t}^{i} \mid A_{t}, w_{t}^{i}, W_{t}, \text { Full Insurance }\right)=\operatorname{Var}\left(m_{t}^{i} \mid A_{t}, w_{t}^{i}, W_{t}\right) .
$$

Hence, if one observes a group of households for which full insurance is satisfied, the degree of risk sharing can be measured for the group for which it is rejected by computing the following statistic:

$$
\xi_{4,1}=\frac{\operatorname{Var}\left(y f_{t}^{i} \mid A_{t}, w_{t}^{i}, W_{t}\right)-\left[\operatorname{Var}\left(\rho_{t}^{i} \mid A_{t}, w_{t}^{i}, W_{t}\right)-\operatorname{Var}\left(\rho_{t}^{i} \mid A_{t}, w_{t}^{i}, W_{t}, \text { Full Insurance }\right)\right]}{\operatorname{Var}\left(y f_{t}^{i} \mid A_{t}, w_{t}^{i}, W_{t}\right)} .
$$

Consider now the case of measurement errors in consumption $m^{i}$ and measurement errors in income $\eta^{i}$. In this case the conditional variance of household income is the sum of the conditional variance generated by the idiosyncratic shocks and of the conditional variance of the measurement errors:

$$
\operatorname{Var}\left(y f_{t}^{i} \mid A_{t}, w_{t}^{i}, W_{t}\right)=\operatorname{Var}\left(f\left(A_{t}, \epsilon_{t}^{i}\right) \mid A_{t}, w_{t}^{i}, W_{t}\right)+\operatorname{Var}\left(\eta_{t}^{i} \mid A_{t}, w_{t}^{i}, W_{t}\right) .
$$

If the measurement errors of consumption and income are drawn from the same distribution, the conditional variance of $\eta^{i}$ corresponds to the conditional variance of $m^{i}$. The degree of risk sharing can therefore be computed using the conditional variance of expenditure for the households that share risk efficiently. Specifically, one can use on the following equation:

$$
\xi_{4,2}=\frac{\operatorname{Var}\left(y f_{t}^{i} \mid A_{t}, w_{t}^{i}, W_{t}\right)-\operatorname{Var}\left(\rho_{t}^{i} \mid A_{t}, w_{t}^{i}, W_{t}\right)}{\operatorname{Var}\left(y f_{t}^{i} \mid A_{t}, w_{t}^{i}, W_{t}\right)-\operatorname{Var}\left(\rho_{t}^{i} \mid A_{t}, w_{t}^{i}, W_{t}, \text { Full Insurance }\right)} .
$$

If they are drawn from a different distribution, however, either the data provide a way of separating the conditional variance of the idiosyncratic shocks from the conditional variance of the measurement errors or one must use $\xi_{4,1}$ as a lower bound of the degree of risk sharing.

Before concluding this section, four remarks are in order. First, if one wishes to measure nonparametrically the degree of risk sharing only the idiosyncratic shocks provide information on it. The 
aggregate shocks provide no additional information since they are allocated to different households according to their risk preferences and decision power, which are unknown and can only be estimated under parametric assumptions and a specific null hypothesis. Second, the computation of the degree of risk sharing requires the estimation of the conditional variance of full income and expenditure. This quantities can be estimated using standard non-parametric methods. In the empirical part, we will employ a kernel estimator. Third, generally the distribution of the idiosyncratic shocks depends on the realization of the aggregate shock. As a consequence, $\operatorname{Var}\left(y f_{t}^{i} \mid A_{t}, w_{t}^{i}, W_{t}\right)$ and $\operatorname{Var}\left(\rho_{t}^{i} \mid A_{t}, w_{t}^{i}, W_{t}\right)$ will also depend on the realization of the aggregate shock. In the empirical part, we will evaluate these quantities at the median of the aggregate shock. Lastly, the approach proposed in this section has some advantages and some limitations. There are two main advantages. The first one is that the method is fully non-parametric and hence it does not rely on functional form assumptions that are required if one uses a model. The second advantage is that one can measure the degree of risk sharing independently of the null hypothesis. The non-parametric specification of the test, however, comes at a price. To compute the degree of risk sharing, one has to construct a measure of the aggregate shock which is not observed. In the empirical part, we will use average resources for the group under consideration to approximate the aggregate shock. In addition, if the measurement errors are a potential problem, one needs to observe a group of households for which full insurance is satisfied. In the empirical part, we will use the households for which efficiency is not rejected in its place. The two limitations just described imply that the test statistic $\xi_{4}$ cannot replace the formal tests of efficient risk sharing developed in this paper. It can only provide evidence on the extend of risk sharing that characterize households for which efficiency is rejected.

\section{Econometric Issues}

In this section we will discuss the econometric issues one has to address to implement the tests. The discussion will be informal and meant to provide some insight. The technical details are described in Appendix B. The results of a simulation study that evaluates the performance of the tests are reported in Appendix C.

\subsection{Estimation of the Risk-sharing Functions}

In the presence of unobservable heterogeneity, the testable implications derived in Section 4 can be used to set up tests of homogeneity in risk preferences and full insurance only if estimates of the household risk-sharing functions are available. In this subsection we discuss the method used to estimate them.

The estimation approach must allow for differences in risk preferences across households. In an environment with different preferences, there are two features of the risk-sharing functions that must 
be considered. First, different households have expenditures with different functional forms. To address this issue we will estimate the risk-sharing functions separately for each household. Second, there is no close form solution for the risk-sharing functions except for the frequently criticized case of CARA utilities. For this reason we will employ a semi-parametric estimator.

Without data limitations, the risk-sharing functions can be estimated non-parametrically separately for each household without making additional assumptions. The dataset employed in this paper has, however, some limitations that will be discussed in the next section. To overcome them, we will impose four restrictions on the way observable and unobservable heterogeneity and measurement errors enter the risk-sharing functions. First, we will assume that the observable and unobservable heterogeneity enter the risk-sharing functions only through the single index $d^{i, j}=\theta_{i, j}\left(z^{i}-z^{j}\right)+\eta^{i}-\eta^{j}$, i.e. only the linear combination of the differences in the heterogeneity variables affects $\rho^{k}$. This assumption simplifies significantly the estimation since all the variation in heterogeneity is summarized by a single variable. Second, it is assumed that the unobservable component of heterogeneity does not change over time. Under this assumption, $\eta^{i}-\eta^{j}$ captures unobservable preference shocks that are very persistent. ${ }^{16}$ As a third assumption, we will impose the restriction that the coefficients on the observable heterogeneity variables are common across households to increase the precision of our estimates. Finally, we will allow for measurement errors $\hat{m}$ in individual expenditure, which are assumed to be multiplicative and independent of $w^{i}, w^{j}, d^{i, j}$. Under these assumptions, the risk-sharing function of household $k$ can be written as follows: ${ }^{17}$

$$
\rho^{k}=\hat{g}^{k}\left(\rho^{i, j}, w^{i}, w^{j}, d^{i, j}\right) \hat{m}^{k} .
$$

We can then take the natural logarithm to obtain a function that is linear in the measurement errors:

$$
\ln \rho^{k}=g^{k}\left(\rho^{i, j}, w^{i}, w^{j}, d^{i, j}\right)+m^{k} .
$$

where $g^{k}$ and $m^{k}$ are the natural logarithm of $\hat{g}^{k}$ and $\hat{m}$. It is important to remark that, since the natural logarithm is a monotonic function, all the testable implications derived in Section 4 hold if one uses $g^{k}$ in place of $\hat{g}^{k}$. In the rest of the paper, we will use the term risk-sharing function to denote $g^{k}$.

Three issues are worth a discussion. First, there is a large class of utilities that generates the risk-sharing function described in (4). For instance, all the utility functions that can be written in the following form:

$$
u^{i}\left(c^{i}, l^{i}, z^{i}, \eta^{i}\right)=v^{i}\left(c^{i}, l^{i}\right) \exp \left(\theta z^{i}+\eta^{i}\right)
$$

produce risk-sharing functions consistent with (4). Second, in the model $\rho^{i, j}$ is equal to the sum of individual expenditures. In the estimation $\rho^{i, j}$ will be computed using this relationship. As a

\footnotetext{
${ }^{16}$ One can allow for a term $\eta^{i}-\eta^{j}$ that varies over time using the estimator proposed by Blundell and Powell (2001). In this case, the risk-sharing function must be replaced by its expectation taken over $\eta^{i}-\eta^{j}$. This generalization is left for future research.

${ }^{17}$ The time subscript will be suppressed here and in the remaining sections to simplify the notation.
} 
consequence, if the data on individual expenditure are affected by measurement errors, the variable $\rho^{i, j}$ will also be affected by the same problem. In the empirical part, we will use an estimator that can address this problem. Lastly, the assumptions on observable and unobservable heterogeneity are needed only if one is interested in the economic meaning of $z$ and $\eta$ and in whether an economic model can generate the particular functional form imposed on the risk-sharing functions. If one is not interested in these issues, observable and unobservable heterogeneity can be introduced by simply adding a polynomial in $z$ to $g^{k}\left(\rho_{t}^{i, j}, w_{t}^{i}, w_{t}^{j}\right)$.

Under the assumptions listed above, the risk-sharing functions can be estimated using the combination of two estimators available in the non-parametric literature. The first one is the estimator developed by Newey et al. (1999). For a given single index $d^{i, j}$ it enables us to estimate nonparametrically the risk-sharing functions controlling for the endogeneity of $\rho_{t}^{i, j}$. The second one is the estimator proposed by Ichimura (1993), which will be used to estimate the parameters of the single index $d^{i, j}$.

\subsection{The Tests}

We will now discuss how the tests of preference homogeneity and full insurance can be implemented using the testable implications derived in Section 4. The discussion will be divided into two parts. In the first part, we will describe how one can derive test statistics for each pair of households in the data. These test statistics can be used to evaluate preference heterogeneity and efficiency separately for each observed pair. The final goal, however, is to set up tests that can reject the null hypotheses for the entire group of households. In the second part of this subsection, we discuss how the test statistics computed for each pair can be combined to construct these tests.

We will start with the the test of homogeneity in risk preferences for a pair of households. It is based on Proposition 3, which states that under efficiency if two households have identical risk preferences their risk-sharing functions should not cross. To simplify the notation we will suppress the dependence of the risk-sharing functions on wages, observable and unobservable heterogeneity.

The test can be constructed using the following idea. Consider the pair composed of households $i$ and $j$, suppose that they share risk efficiently, and denote by $g^{i, j}$ the difference in their risk-sharing functions. Under the null of identical preferences, $g^{i, j}$ as a function of aggregate resources should always be either positive or negative since there cannot be a crossing. As a consequence, the area below the positive part of $g^{i, j}$ multiplied by the area above the negative part of $g^{i, j}$ is equal to zero under the null, but it is positive under the alternative. Formally,

$$
\xi_{1}^{i, j}=-\left(\int_{\left\{u: g^{i, j}(u) \geq 0\right\}} g^{i, j}(u) d u\right)\left(\int_{\left\{u: g^{i, j}(u)<0\right\}} g^{i, j}(u) d u\right)\left\{\begin{array}{ll}
=0 & \text { under } H_{0} \\
>0 & \text { under } H_{A}
\end{array} .\right.
$$

Thus, if $\xi_{1}^{i, j}$ is positive the null hypothesis of homogeneous risk preferences can be rejected. ${ }^{18}$

\footnotetext{
${ }^{18}$ We have also experimented with the simpler test statistic $\bar{\xi}^{i, j}=\max \left\{g^{i, j}\right\} \min \left\{g^{i, j}\right\}$. Our simulation results,
} 
Using this idea the test can be implemented in three steps. In the first step, the difference between the risk-sharing functions is estimated using the method discussed in the previous subsection. In the second step, the test statistic $\hat{\xi}_{1}^{i, j}$ is computed by using the sample analog of (5). In the final step, the distribution of $\hat{\xi}_{1}^{i, j}$ is recovered by bootstrap. The null is then rejected for the pair composed of households $i$ and $j$ if the estimated test statistic $\hat{\xi}_{1}^{i, j}$ is statistically too large.

We will now describe the implementation of the first test of full insurance. It is based on the first testable implication of Proposition 4 according to which any variable that captures idiosyncratic shocks should not enter the risk-sharing functions. This implication is tested by employing the semiparametric test of omitted variables proposed by Fan and Li (1996), which is described in Appendix B. Specifically, we first compute the test statistic derived by Fan and Li (1996). We then bootstrap its distributions and reject the null for the pair of households under investigation if the test statistic is statistically too large.

The second test of full insurance is based on the implication that under efficiency the risk-sharing functions should increase with total resources. This implication is tested using the monotonicity test introduced by Hall and Heckman (2000), the details of which are provided in Appendix B. As for the previous two tests, we first compute the test statistic introduced by Hall and Heckman (2000). Its distribution is then recovered by bootstrap. Finally, full insurance is rejected if the test statistic is statistically too large.

In the last part of this subsection, we explain how the test statistics at the pair level can be combined to obtain one test of preference homogeneity and two tests of efficiency with the following two features. First, each null hypothesis is tested simultaneously for all households in the group under investigation. Second, if the null is rejected, the tests enable us to determine the fraction of households for which the hypothesis is rejected. The tests at the group level are based on the multiple testing procedure developed in Romano and Wolf (2005) and Romano, Shaikh, and Wolf (2006). ${ }^{19}$ Consider $n$ hypotheses $H_{1}, \ldots, H_{n}$ and let $T_{1}, \ldots, T_{n}$ be the associated test statistics. Suppose that one is interested in a null hypothesis $H_{0}$ which is equal to the intersection of $H_{1}, \ldots, H_{n}$, in the sense that $H_{0}$ is not rejected only if each individual hypothesis $H_{k}$ is not rejected. Romano and Wolf (2005) and Romano, Shaikh, and Wolf (2006) propose two methods for testing $H_{0}$. The first method controls the familywise error rate (FWE), i.e. the probability of rejecting at least one of the true hypotheses. Romano, Shaikh, and Wolf (2006) provide evidence that if the number of individual hypotheses is large, the method that controls for the FWE rate is too conservative. They propose an alternative method called $k$-stepM method which controls the $k$-FWE rate, i.e. the probability of rejecting at least $k$ true hypotheses. Since in our paper the number of individual hypotheses is between 1 and

however, suggest that a test based on $\bar{\xi}^{i, j}$ has less power and control than a test based on $\xi^{i, j}$. The difference in power and control is especially large in the case of measurement errors with high variance.

${ }^{19}$ There are other procedures that enable one to test multiple hypotheses. See for instance Holm (1979), Hochberg and Tamhane (1987), and Hommel (1988). The advantage of the methods proposed by Romano and Wolf (2005) and Romano, Shaikh, and Wolf (2006) is that they allow for dependence between the individual hypotheses. 
557 , we adopt the $k$-stepM method. This method, which is described in details in Appendix B, can be implemented in three steps. First, the $n$ test statistics must be ordered from the highest to the lowest. Second, one has to compute the $k$-th largest test statistic and the $1-\alpha$ percentile of its sampling distribution, where $\alpha$ is the desired significance level. Third, a confidence region for the $n$ hypotheses must be constructed using the $1-\alpha$ percentile. An individual hypothesis is then rejected if it is outside the confidence region. The process is repeated with the set of individual hypotheses that were not rejected until the number of rejections is smaller than $k$.

\section{The ICRISAT Dataset}

In the rest of the paper we use the three tests developed here jointly with the Village Level Studies (VLS) started by the ICRISAT to understand risk sharing in rural India. This dataset has been chosen for two reasons. First, a good understanding of the effect of idiosyncratic and aggregate shocks on household welfare is particularly important in developing countries, where shocks may have devastating effects on household resources because of the small number of formal markets. Second, several papers in the past have tested efficient risk sharing using this dataset. The results can therefore be compared with the findings of previous papers.

The ICRISAT started the VLS at six locations in rural India on July 1975. The study added four villages in 1981. In each village 40 households were selected to represent families in four land holding classes: 10 from landless laborers; 10 from small farmers; 10 from medium farmers; 10 from large farmers. Following Townsend (1994), the sample used in the estimation is composed of households from 3 villages: Aurepalle, Shirapur, and Kanzara. The VLS records data on production, labor supply, assets, price of goods, rainfall, monetary and non-monetary transaction, household size, age, education, and three different caste rankings from 1975 to 1985. Townsend (1994) gives a detailed description of the data. We will therefore discuss only the issues that are specific to our paper.

In the estimation we need data on consumption, labor supply, wages, demographic variables, and non-labor income. The ICRISAT collects information on these variables approximately every month. The risk-sharing functions can therefore be estimated using monthly data. We will now discuss how these variables are constructed.

Monthly household consumption is calculated using the transaction data from the ICRISAT Household Transaction Schedule. The consumption variable is the sum of consumption of milled grain, consumption of other food items, namely oil, animal products, fruits and vegetables, and consumption on other non-durable goods, which is composed of electricity, water charges, cooking fuels for household use, and expenses for domestic work. There are two main problems with using the transaction files: the frequency of the interviews varies; the dates of the interviews differ across households. For example, a household in Aurepalle was interviewed on January 11, February 10, and March 21 in 1980, whereas a different household in the same village was interviewed on January 17, February 13, 
and March 25 in the same year. It is therefore difficult to compare expenditures across households and over time. To overcome this problem for each interview that covers two months we compute the percentage of days that belongs to each month. We then assign to each month the corresponding expenditure using this percentage. ${ }^{20}$ The measure of consumption that we obtain using this method corresponds to non-durable consumption for the entire household. Since different households have different size and gender-age structure, we divide household consumption by an age-gender weight which is constructed following Townsend (1994). ${ }^{21}$

The construction of the wage and labor supply variables requires a separate discussion. Three different types of employment and wages are recorded by the ICRISAT. The Labor, Draft Animal, and Machinery Utilization Schedule contains information on hours, days of employment, and wages of individuals entering daily employment outside their own farm. In the Household Transaction Schedule, labor income of individuals with regular jobs outside their own farm is recorded, but there is no information on the days and hours of employment. We assume that the data on regular labor income refer to the period covered by the interview and that the individual with the regular job works 8 hours a day for 5 days a week. In the Plot Cultivation Schedule, the ICRISAT records data on the number of hours supplied by men, women, and children to their own farm and the value of their labor. The value of own labor is imputed by the ICRISAT on the basis of the village-specific market wages. The data in these three schedules are collected every interview. We employ this information to construct the daily wages and labor supply that correspond to each month using the method described for consumption. Daily labor supply is therefore the average number of hours of employment on daily jobs, regular jobs, and jobs on own farm supplied by adult members. Daily wages are the average of total labor income earned on any job by adult members divided by the total number of hours. In the construction of leisure we compute the time endowment $T$ by assuming that each individual has 26 days per month and 16 hours per day that can be divided between labor and leisure. The remaining days and hours account for sleep, sickness, and holidays. The first full insurance test is implemented using non-labor income as the omitted variable. It is constructed as the sum of income from gifts, dowries, pension, theft, and profits.

\footnotetext{
${ }^{20}$ As an alternative, we could have assumed a fixed daily rate for each month and then assign expenditure accordingly. This method has two main problems. First, there are several daily rates that are consistent with the observed data depending on which of the two months one starts with. Second, in many cases the daily rate and therefore expenditure is negative. For instance, consider a household with a first interview that covers the period January 1 to January 21 , a second one that covers the period January 22 to February 10, and a third interview that covers the period February 11 to February 28. Suppose that expenditure is 100 in the first interview, 45 in the second one, and 90 in the third one. Using the first interview one can find that the fixed daily rate for January is $100 / 21=4.8$. Using this rate and the second interview we can then determine the fixed daily rate for February. The expenditure for the first 10 days of February must be $45-4.8^{*} 10=-3$, which implies that the daily rate for February must be -0.3 and monthly expenditure -8.4. Observe also that the monthly expenditure for January and February can be computed starting from the third interview. In this case, the daily rate for February must be $90 / 18=5$ and expenditure $5 * 28=140$. Using the second interview the rate for January can be computed to be $45-5 * 10=-5$. Hence, if we start with the third interview we have different expenditures and a negative expenditure for January. Because of these two problems we have decided to use the approach described above.

${ }^{21}$ The age-gender weight is computed by adding the following numbers: for adult males, 1.0; for adult females, 0.9; for males aged 13-18, 0.94; for females aged 13-18, 0.83; for children aged 7-12, 0.67; for children aged 4-6, 0.52; for Toddlers 1-3, 0.32; and for infants 0.05 .
} 
The Households Member Schedule records data on demographic variables. We use this information to construct the vector of observable heterogeneity variables, which is composed of the mean age of adult household members, the number of infants, and the age-gender weight. Consumption and wages are deflated using the consumer price index for agricultural laborers published by the Labour Bureau of India. The set of instruments used to address the endogeneity of aggregate resources is constructed using information on lagged rainfall, lagged total expenditure, and lagged savings. When we divide the sample in different castes we use the caste rank considered in Behrman (1988). It is constructed using the caste rank prepared by a social anthropologist which is based on social, religious, and economic standing in the village.

In the estimation of the degree of risk sharing for households for which efficiency is rejected, two additional variables are required: household full income, $y f_{t}^{i}$, and a variable that measures the aggregate shock, $A_{t}$. Using the household budget constraint, full income is computed as total expenditure minus the resources borrowed from different sources, plus the resources saved in different accounts or lent to various individuals, plus the transfers given out, minus the transfers received, plus taxes. The ICRISAT data contains information on all these variables except one. We do not observe the amount of cash saved and not deposited in a financial institution. Our measure of risk sharing is therefore likely to underestimate the degree of risk sharing. As a measure of the aggregate shock we use average full income for the group of households that is being considered.

The sample period is July 1975 to July 1985 for Aurepalle and July 1975 to July 1984 for Shirapur and Kanzara. We drop the households that leave the sample before 1985 for Aurepalle and 1984 for Shirapur and Kanzara. This implies that we have up to 126 observations for each household in Aurepalle and up to 114 observations for each household in Shirapur and Kanzara. In all tests, we drop a household if it has fewer than 80 data points. ${ }^{22}$ Table 1 reports the summary statistics of the main variables.

\section{Results}

In this section, we report the outcome of the tests for the three Indian villages considered in the paper. We will first describe the outcome of the test of homogeneity in risk preferences. The estimation of the household risk-sharing functions indicates that every pair of households can be assigned to one of three different categories: (i) pairs whose risk-sharing functions do not cross; (ii) pairs with risk-sharing functions that cross once; (iii) pairs whose risk-sharing functions cross twice. The first two panels of Figure 5 depict one pair of households for the first two categories. Figure 6 describes the risk-sharing functions for a pair in the third category. This finding represents a first and informal indication that heterogeneity in risk preferences is a significant feature of Indian villages. The outcome of the formal

\footnotetext{
${ }^{22}$ By including households that have data only on some dates, we need to make the assumption that the dates that are not observed are missing at random with respect to the measurement errors.
} 
test, which is reported in Table 2, supports this evidence. At the village level, homogeneity in risk preferences is strongly rejected in all three villages. We reject the null in $32 \%$ of possible cases in Aurepalle, in $25 \%$ of possible cases in Shirapur, and in $25 \%$ of possible cases in Kanzara. We then perform the test at the caste level by considering the households in a given village that belong to the same caste. In this case, we reject the hypothesis of identical risk preferences in eight of the thirteen observed castes. These results imply that previous tests should have rejected efficient risk sharing in Indian villages as long as aggregate shocks have a significant impact on household behavior and the variable used to capture idiosyncratic shocks is correlated with risk preferences.

We will now discuss the outcome of the efficiency tests. Before considering the tests proposed in this paper, we describe the results obtained using the standard test employed in the risk-sharing literature. The results, which are reported in Table 3, are obtained using the specification proposed by Townsend (1994). It corresponds to the following efficiency condition:

$$
\Delta \rho_{t}^{i}=\alpha_{0}+\alpha_{1} \Delta X_{t}+\alpha_{2} \Delta \rho_{t}^{a}+\alpha_{3} \Delta y_{t}^{i}
$$

where $\Delta \rho_{t}^{i}, \Delta \rho_{t}^{a}$, and $\Delta y_{t}^{i}$ are the first differences in household expenditure, village expenditure, and household income, and $X_{t}$ is a vector of control variables that includes wages, mean household age, age-gender weight, and number of infants. To be able to compare the results of the standard test with the results of the tests developed in the present paper, expenditure is defined as expenditure on non-durable consumption plus expenditure on leisure. We reject efficiency in all three villages, which is consistent with the results reported in Townsend (1994). The test is also performed at the caste level for castes that have at least two households in the sample. In this case $\Delta \rho_{t}^{a}$ measures the first difference in caste expenditure. In all three villages, we reject full insurance for all castes. ${ }^{23}$

We will now describe the outcome of the two efficiency tests derived in this paper. The results are reported in Table 4. At the village level, both tests reject efficient risk sharing in all three villages. Using the test based on increasing functions, efficiency is rejected for three pairs in Aurepalle, nine pairs in Shirapur, and six pairs in Kanzara. These numbers correspond to about $1 \%$ of possible cases in Aurepalle, $2 \%$ of possible cases in Shirapur, and $1 \%$ of possible cases in Kanzara. ${ }^{24}$ The results obtained using the efficiency test based on non-labor income are consistent with the ones obtained using increasing functions. To summarize, at the village level we observe a small number of rejections, which implies that there is a significant degree of risk sharing in Indian villages. But the violations of the testable implications are sufficiently large that the tests can detect a rejection of full insurance even if one allows for heterogeneity in risk preferences and a general class of utility functions.

Allowing for heterogeneity in risk preferences and a general class of utility functions makes a significant difference when we test risk sharing at the caste level. Contrary to the outcome of the standard

\footnotetext{
${ }^{23}$ This paper is not the first one to reject full insurance at the caste level using the standard test. Morduch (2004) find similar results using the ICRISAT data.

${ }^{24}$ Observe that our tests control the k-FWE rate. This implies that in our tests the probability of rejecting at least $k$ true hypotheses is controlled to be smaller than or equal to 0.05 . The statement that the tests should reject at least $5 \%$ of total hypotheses is therefore incorrect in this context.
} 
test, we find that full insurance represents a good approximation of the behavior of households that belong to the same caste. In Aurepalle and Shirapur we never reject full insurance. Kanzara is the only village in which we observe one caste for which efficiency is rejected. For this caste, the null is rejected for one pair out of 78 possible cases.

One additional point should be discussed. For the castes with ranking 30, 86.25, and 97.5 in Aurepalle, with ranking 5 in Shirapur, and with ranking 11.25 in Kanzara we cannot reject the hypothesis of homogeneity in risk preferences, we reject efficiency using the standard test, but we cannot reject efficient risk sharing using the semi-parametric tests that allow for heterogeneity in risk preferences. In these cases the different outcome should be attributed to the general class of utility functions allowed by our tests and not to the presence of heterogeneous risk preferences. ${ }^{25}$

We will now discuss the degree of risk sharing that characterizes the households for which full insurance is rejected using the method developed in Section 5. Since the outcome of the efficiency tests suggests that the caste is the proper risk-sharing unit, we have computed the degree of risk sharing at the caste level using as the aggregate shock average full income for the caste under consideration. We present the results obtained by controlling for measurement errors in consumption and income. In Aurepalle and Shirapur, efficiency is never rejected at the caste level. In Kanzara, we reject efficiency for the caste with index 55. The estimated degrees of risk sharing for the thirteen households that we observe for this caste are equal to $38 \%, 50 \%, 58 \%, 62 \%, 70 \%, 70 \%, 75 \%, 76 \%, 77 \%, 89 \%, 92 \%$, $92 \%$, and $99 \%$. In all cases, the values are statistically different from zero. Our measure indicates that this caste can achieve a high level of insurance even if efficiency is rejected. There is only one household for which the share of idiosyncratic shocks it can insurance against is below $50 \%$. All the others can eliminate a significant fraction of the risk they face. This finding confirms the hypothesis that full insurance is an excellent approximation of the choices that characterize the households that belong to the same caste. $^{26}$

The main empirical findings of this paper can be summarized as follows. First, a model with full insurance is a good approximation of the behavior of households that belong to the same caste. Second, efficient risk sharing is rejected at the village level. The number of rejections is small, but there is a non-negligible number of cases in which the data indicate that households that belong to

\footnotetext{
${ }^{25}$ Observe that the differences between the standard test and the new tests proposed here could be due to the new tests having lower power than the standard test. Our simulation study indicates, however, that our tests have good power.

${ }^{26}$ We have also computed the degree of risk sharing for households for which efficiency is not rejected. In this case the measurement errors may affect the results, since we cannot control for them. One should therefore interpret these results with caution. In Aurepalle and Shirapur, where we never reject full insurance at the caste level, we find that castes generally experience a high degree of risk sharing with a median that is equal to $85 \%$ of idiosyncratic shocks in Aurepalle and $87 \%$ in Shirapur. In Kanzara, when we exclude the caste for which efficiency is rejected, we also observe a high degree of risk sharing at the caste level with a median that is equal to $93 \%$. The results are not perfect, however. We observe a couple of households for which the degree of risk sharing is below $50 \%$ even if we do not reject full insurance for their castes. Since our simulation results suggest that our efficiency tests have good power, there are two possible explanations for the low degree of risk sharing for these households. First, a large fraction of their savings is not deposited in a financial institution and hence is not recorded by the ICRISAT. Alternatively, measurement errors are particularly problematic for these households.
} 
different castes are affected by caste-specific shocks. These two findings suggest that the relevant social unit in rural India is not the village, as indicated in previous papers, but the caste. These results are consistent with recent evidence reported in Munshi and Rosenzweig (2009), where it is shown that to understand mobility in India one should use the caste as the social unit.

We will now provide some descriptive evidence about the type or risk-sharing institutions that castes use to insure their members against different types of shocks. A formal analysis would require a precise description of each institution, which is beyond the scope of this paper. Using the ICRISAT data, however, we can give some insight on one institution that appears to be important as a risksharing device at the caste level: transfers. We use two types of information available in the ICRISAT. First, the ICRISAT collects data on the amount received by or given to a household as a transfer. In the ICRISAT questionnaire a transfer is defined as a transaction in which resources or money change ownership without compensation. Second, in the ICRISAT we observe the partner in the transaction, where the list of partners includes caste fellows. We can therefore determine whether a transfer took place between two households that belong to the same caste.

Table 5 reports average real per-capita transfers given by and received from households in the three villages under investigation. There are three patterns that are worth a discussion. First, transfers are a substantial fraction of non-durable expenditure. In Aurepalle, real per-capita transfers given and received are on average $28.3 \%$ and $21.1 \%$ of non-durable expenditure. In Shirapur, the transfers given and received are also a high percentage of expenditure at $16.0 \%$ and $21.0 \%$. The village with the lower amount of transfers is Kanzara where transfers given and received are $8.9 \%$ and $15.9 \%$. A second pattern worth describing is that most of the transfers are given to and received from households that belong to the same caste. In Aurepalle, the transfers given to caste fellows are $87 \%$ of total transfers and the transfers received from caste fellows are $68 \%$. Shirapur and Kanzara are characterized by similar percentages. These findings are consistent with Townsend's view that transfers are an important source of insurance. A last feature of the data that should be discussed is that a large fraction of the transfers between caste fellows is given to and received from households living outside the village boundaries. This pattern is consistent with previous findings, see for instance Rosenzweig and Stark (1989), which indicate that the relationship between caste members extends beyond the village.

We have also analyzed the data on loans received from and given to other households. Our findings are reported in the second part of Table 5. Similarly to the transfers, the average value of loans given as well as received are a significant fraction of household expenditure, with the loans received being a substantially higher fraction than the loans given. Some of the loans are given to and received from caste fellows. The majority of them, however, come from and end up outside the caste. 


\section{Conclusions}

In the paper we show that, if in the data households have heterogeneous risk preferences, the standard test of efficient risk sharing rejects efficiency even if households share risk perfectly. To address this problem, we propose a method that enables one to test for efficiency even if risk preferences are heterogeneous. We apply this method to Indian villages. We find strong evidence against the common assumption of identical risk preferences. We also find that efficient risk sharing is rejected in Indian villages, but it is not rejected for the castes that compose those villages. This finding suggests that the insurance policies that are more likely to improve the welfare of individuals living in Indian villages are policies that insure households against aggregate shocks at the caste, village, or state level.

The method proposed in this paper can be used in other environments provided that a long panel is available. For instance, the method can be used to test efficient risk sharing across countries using the long panels that exist at the country level. The method can also be used to test efficient risk sharing in the US at the country, state, extended-family, or household level using the CEX and synthetic cohorts. 


\section{References}

Aiyagari, Rao S., and Mark Gertler. 1991. "Asset returns with transaction costs and uninsured individual risk." Journal of Monetary Economics 27 (3): 311-331 (June).

Altonji, Joseph G., Fumio Hayashi, and Laurence J. Kotlikoff. 1992. "Is the Extended Family Altruistically Linked? Direct Tests Using Micro Data." The American Economic Review 82 (5): 1177-1198 (December).

Altug, Sumru, and Robert A. Miller. 1990. "Household Choices in Equilibrium." Econometrica 58 (3): 543-570 (May).

Amin, Sajeda, Ashok S. Rai, and Giorgio Topa. 2003. "Does microcredit reach the poor and vulnerable? Evidence from northern Bangladesh." Journal of Development Economics 70 (1): 59-82 (February).

Attanasio, Orazio, and Steven J. Davis. 1996. "Relative Wage Movements and the Distribution of Consumption." The Journal of Political Economy 104 (6): 1227-1262 (December).

Attanasio, Orazio, and José Victor Rios-Rull. 2000. "Consumption Smoothing and Extended Families." Manuscript, July.

Behrman, Jere R. 1988. "Intrahousehold Allocation of Nutrients in Rural India: Are Boys Favored? Do Parents Exhibit Inequality Aversion?" Oxford Economic Papers 40 (1): 32-54 (March).

Blundell, Richard, Luigi Pistaferri, and Ian Preston. 2006. "Consumption Inequality and Partial Insurance." IFS Working Paper.

Blundell, Richard, and James L. Powell. 2001. "Endogeneity in Nonparametric and Semiparametric Regression Models." Cemmap Working Paper.

Cochrane, John H. 1991. "A Simple Test of Consumption Insurance." The Journal of Political Economy 99 (5): 957-976 (October).

Dubois, Pierre. 2004. "Consumption Insurance with Heterogeneous Preferences. Can Sharecropping Help Complete Markets?" Manuscript, University of Toulouse.

Fan, Yanqin, and Qi Li. 1996. "Consistent Model Specification Tests: Omitted Variables and Semiparametric Functional Forms." Econometrica 64 (4): 865-890 (July).

Fuchs-Schundeln, Nicola, and Matthias Schundeln. 2005. "Precautionary Savings and Self-Selection: Evidence from the German Reunification 'Experiment'." Quarterly Journal of Economics 120 (3): 1085-1120 (August).

Hall, Peter, and Nancy E. Heckman. 2000. "Testing for Monotonicity of a Regression Mean by Calibrating for Linear Functions." The Annals of Statistics 28 (1): 20-39 (February).

Ham, John, and Kris Jacobs. 2000. "Testing for Full Insurance Using Exogenous Information." Journal of Business and Economic Statistics 18 (4): 387-397 (October).

Hara, Chiaci. 2006. "Necessary and Sufficient Conditions for the Efficient Risk-Sharing Rules and the Representative Consumer's Utility Function." Manuscript, Kyoto University. 
Hara, Chiaci, James Huang, and Christoph Kuzmics. 2006. "Efficient Risk-Sharing Rules with Heterogeneous Risk Attitudes and Background Risks." Manuscript, KIER Discussion Paper Series.

Hayashi, Fumio, Joseph Altonji, and Laurence Kotlikoff. 1996. "Risk-Sharing between and within Families." Econometrica 64 (2): 261-294 (March).

Heathcote, Jonathan, Kjetil Storesletten, and Gianluca Violante. 2007. "Consumption and Labor Supply with Partial Insurance: An Analytical Framework." Manuscript.

Hicks, John R. 1990. "Value and Capital." Oxford: Oxford University Press.

Hochberg, Yosef, and Ajit C. Tamhane. 1987. Multiple Comparisons Procedures. New York: Wiley.

Holm, Sture. 1979. "A Simple Sequentially Rejective Multiple Tests Procedure." Scandinavian Journal of Statistics 6 (1): 65-70.

Hommel, Gerhard. 1988. "A Stagewise Rejective Multiple Test Procedure Based on a Modified Bonferroni Test." Biometrika 75 (2): 383-386 (June).

Ichimura, Hidehiko. 1993. "Semiparametric Least Squares (SLS) and Weighted SLS Estimation of Single-Index Models." Journal of Econometrics 58 (1-2): 71-120 (July).

Kocherlakota, Narayana R. 1996. "Implications of Efficient Risk Sharing without Commitment." Review of Economic Studies 63 (4): 595-609 (October).

Kreps, David M. 1990. A Course in Microeconomic Theory.

Krueger, Dirk, and Hanno Lustig. 2010. "When is market incompleteness irrelevant for the price of aggregate risk (and when is it not)?" Journal of Economic Theory 145 (1): 1-41 (January).

Ligon, Ethan. 1998. "Risk Sharing and Information in Village Economies." Review of Economic Studies 65 (4): 847-864 (October).

Ligon, Ethan, Jonathan P. Thomas, and Tim Worrall. 2002. "Informal Insurance Arrangements with Limited Commitment: Theory and Evidence from Village Economies." Review of Economic Studies 69 (1): 209-244 (January).

Lucas, Deborah J. 1994. "Asset pricing with undiversifiable income risk and short sales constraints: Deepening the equity premium puzzle." Journal of Monetary Economics 34 (3): 325-341 (December).

Mace, Barbara J. 1991. "Full Insurance in the Presence of Aggregate Uncertainty." The Journal of Political Economy 99 (5): 928-956 (October).

Mazzocco, Maurizio. 2004. "Savings, Risk Sharing and Preferences for Risk." American Economic Review 94 (4): 1169-1182 (September).

Morduch, Jonathan. 2004. "Consumption Smoothing Across Space: Testing Theories of Risk-Sharing in the ICRISAT Study Region of South India." In: Dercon, S., (Ed.), Insurance Against Poverty. Oxford University Press, Oxford.

Munshi, Kaivan, and Mark Rosenzweig. 2009. "Why is Mobility in India so Low? Social Insurance, Inequality, and Growth." BREAD Working Paper No. 092. 
Newey, Whitney K., James L. Powell, , and Francis Vella. 1999. "Nonparametric Estimation of Triangular Simultaneous Equations Models." Econometrica 67 (3): 565-603 (May).

Ogaki, Masao, and Qiang Zhang. 2001. "Decreasing Relative Risk Aversion and Tests of Risk Sharing." Econometrica 69 (2): 515-526 (March).

Ravallion, Martin, and Shubham Chaudhuri. 1997. "Risk and Insurance in Village India: Comment." Econometrica 65 (1): 171-184 (January).

Romano, Joseph P., Azeem M. Shaikh, and Michael Wolf. 2006. "Formalized Data Snooping Based on Generalized Error Rates." Working Paper, Stanford University.

Romano, Joseph P., and Michael Wolf. 2005. "Stepwise Multiple Testing as Formalized Data Snooping." Econometrica 73 (4): 1237-1282 (July).

Rosenzweig, Mark R., and Oded Stark. 1989. "Consumption Smoothing, Migration, and Marriage: Evidence from Rural India." The Journal of Political Economy 97 (4): 905-926 (August).

Schmidt, Klaus D. 2003. "On the Covariace of Monotone Functions of a Random Variable." Manuscript, University of Dresden.

Schulhofer-Wohl, Sam. 2010. "A Test of Consumption Insurance with Heterogeneous Preferences." Manuscript, Princeton University.

Townsend, Robert M. 1994. "Risk and Insurance in Village India." Econometrica 62 (3): 539-591 (May). 


\section{A Proofs}

In all the proofs we will suppress the dependence on observable and unobservable heterogeneity to simplify the notation.

\section{A.1 Proof of Proposition 1}

Proof. If the preferences belong to the HARA class, $f\left(c_{t}^{i}\right)=\ln \left(a+c_{t}^{i}\right)$. Consequently the regression equation (2) can be written as

$$
\Delta \ln \left(a+c_{t}^{i}\right)-\frac{1}{n} \sum_{j=1}^{n} \Delta \ln \left(a+c_{t}^{j}\right)=\xi \Delta y_{t}^{i}+\epsilon_{t},
$$

The heterogeneity in the risk aversion parameter $\gamma$ implies that there is an omitted variable from equation (6). If this variable is denoted by $e_{t}^{i}$, the equation can be rewritten in the following way:

$$
\Delta \ln \left(a+c_{t}^{i}\right)-\frac{1}{n} \sum_{j=1}^{n} \Delta \ln \left(a+c_{t}^{j}\right)=\xi \Delta y_{t}^{i}+e_{t}^{i}+\eta_{t}
$$

where $E\left[\eta_{t}\right]=0$ and $\eta_{t}$ is independent of the aggregate shocks, the idiosyncratic shocks, and the risk aversion parameters. Standard results on omitted variable biases imply that

$$
E(\hat{\xi})=\xi+\operatorname{VAR}\left(\Delta y_{t}^{i}\right)^{-1} \operatorname{Cov}\left(\Delta y_{t}^{i}, e_{t}^{i}\right)
$$

where the expectations are generally taken over the risk aversion parameters, the idiosyncratic, and the aggregate shocks. Efficient risk sharing implies that $\xi=0$. Hence, $E(\hat{\xi})>0$ if $\operatorname{Cov}\left(\Delta y_{t}^{i}, e_{t}^{i}\right)>0$.

Observe that $\Delta y_{t}^{i}$ depends on both the idiosyncratic and the aggregate shocks, whereas under full insurance $e_{t}^{i}$ depends only on the aggregate shocks. Let $E_{\gamma, A, I}$ be the expected value operator computed with respect to the joint distribution of the risk aversion parameter, the aggregate shock, and the idiosyncratic shock. $E_{A, I}, E_{\gamma, A}, E_{A}, E_{I}$, and $\operatorname{Cov}_{\gamma}$ are similarly defined. Then the law of iterated expectations implies that the following equalities are satisfied:

$$
\begin{aligned}
\operatorname{Cov}\left(\Delta y_{t}^{i}, e_{t}^{i}\right) & =E_{\gamma, A, I}\left[\Delta y_{t}^{i} e_{t}^{i}\right]=E_{\gamma}\left[E_{A, I}\left[\Delta y_{t}^{i} e_{t}^{i} \mid \gamma\right]\right]=E_{\gamma}\left[E_{A}\left[E_{I}\left[\Delta y_{t}^{i} \mid \gamma, A\right] e_{t}^{i} \mid \gamma\right]\right]= \\
& =E_{\gamma, A}\left[E_{I}\left[\Delta y_{t}^{i} \mid \gamma, A\right] e_{t}^{i}\right]=E_{A}\left[E_{\gamma}\left[E_{I}\left[\Delta y_{t}^{i} \mid \gamma, A\right] e_{t}^{i} \mid A\right]\right]= \\
& =E_{A}\left[\operatorname{Cov}_{\gamma}\left(E_{I}\left[\Delta y_{t}^{i} \mid \gamma, A\right], e_{t}^{i} \mid A\right)\right],
\end{aligned}
$$

where the second, third, fourth, and fifth equalities follow from the low of iterated expectations. As a consequence, $E(\hat{\xi})>0$ if $\operatorname{Cov}_{\gamma}\left(E_{I}\left[\Delta y_{t}^{i} \mid \gamma, A\right], e_{t}^{i} \mid A\right)>0$ for each realization of the aggregate shocks. We will show that this inequality holds using the following result: ${ }^{27}$

$$
\operatorname{Cov}(f(x), g(x))>0 \quad \text { if } f(x) \text { and } g(x) \text { are increasing functions. }
$$

${ }^{27}$ For a proof see Schmidt (2003). 
Observe that by assumption, for a given realization of the aggregate shocks, $E_{I}\left[\Delta y_{t}^{i} \mid \gamma, A\right]$ is decreasing in $\gamma_{i}$ if $\Delta Y_{t} \geq 0$ and increasing if $\Delta Y_{t}<0$. Hence, $\operatorname{Cov}_{\gamma}\left(E_{I}\left[\Delta y_{t}^{i} \mid \gamma, A\right], e_{t}^{i} \mid A\right)>0$ if $e_{t}^{i}$ is decreasing in $\gamma_{i}$ if $\Delta Y_{t} \geq 0$ and increasing if $\Delta Y_{t}<0$. We will now show that this is the case.

For HARA preferences with heterogeneous $\gamma$, for any realization of $A$ the first order condition of the Pareto problem implies

$$
\ln \left(a+c_{t}^{i}\right)=-\frac{1}{\gamma_{i}} \ln \left(\lambda_{t}\right)+\frac{1}{\gamma_{i}} \ln \left(\mu^{i}\right) .
$$

where $\lambda_{t}$ is the multiplier of the feasibility condition at $t$. Hence,

$$
\Delta \ln \left(a+c_{t}^{i}\right)=-\frac{1}{\gamma_{i}} \Delta \ln \left(\lambda_{t}\right) .
$$

Summing the above equation over all households and dividing by $n$ we obtain,

$$
\begin{aligned}
\frac{1}{n} \sum_{j} \Delta \ln \left(a+c_{t}^{j}\right) & =-\frac{1}{n} \sum_{j} \frac{1}{\gamma_{j}} \Delta \ln \left(\lambda_{t}\right) \\
& =-\frac{1}{\bar{\gamma}} \Delta \ln \left(\lambda_{t}\right)
\end{aligned}
$$

where $\bar{\gamma}=\left(\frac{1}{n} \sum_{j} \frac{1}{\gamma_{j}}\right)^{-1}$ is the harmonic mean of the risk aversion parameters. The regression equation (7) implies that for any realization of $A$

$$
e_{t}^{i}=\Delta \ln \left(a+c_{t}^{i}\right)-\frac{1}{n} \sum_{j} \Delta \ln \left(a+c_{t}^{j}\right)-\eta_{t} .
$$

Substituting for $\Delta \ln \left(a+c_{t}^{i}\right)$ and $\frac{1}{n} \sum_{j} \Delta \ln \left(a+c^{j}\right)$ using equations (9) and (10), $e_{t}^{i}$ can therefore be written as follows:

$$
e_{t}^{i}=\left(\frac{1}{\bar{\gamma}}-\frac{1}{\gamma^{i}}\right) \Delta \ln \left(\lambda_{t}\right)+\eta_{t}
$$

Since $\eta_{t}$ is independent of the risk aversion parameters, the derivative of $e_{t}^{i}$ with respect to $\gamma_{i}$ can be written as

$$
\frac{\partial e_{t}^{i}}{\partial \gamma_{i}}=\Delta \ln \left(\lambda_{t}\right) \frac{n-1}{n} \frac{1}{\left(\gamma^{i}\right)^{2}}
$$

where $n$ is the number of households in the economy.

Finally, it is straightforward to show by differentiating the first order condition (8) with respect to $Y_{t}$ that $\ln \left(\lambda_{t}\right)$ is decreasing in $Y_{t}$. Consequently, $\Delta \ln \left(\lambda_{t}\right)>0$ if $\Delta Y_{t}<0$ and $\Delta \ln \left(\lambda_{t}\right) \leq 0$ if $\Delta Y_{t} \geq 0$. From (11), we can therefore conclude that $e_{t}^{i}$ is decreasing in $\gamma_{i}$ if $\Delta Y_{t} \geq 0$ and increasing if $\Delta Y_{t}<0$. Hence,

$$
\begin{aligned}
\operatorname{Cov}_{\gamma}\left(E_{I}\left[\Delta y_{t}^{i} \mid \gamma, A\right], e_{t}^{i} \mid A\right) & =\operatorname{Cov}_{\gamma}\left(E_{I}\left[\Delta y_{t}^{i} \mid \gamma, A\right], e_{t}^{i} \mid A, \Delta Y_{t} \geq 0\right) P\left(\Delta Y_{t} \geq 0\right) \\
& +\operatorname{Cov}_{\gamma}\left(E_{I}\left[\Delta y_{t}^{i} \mid \gamma, A\right], e_{t}^{i} \mid A, \Delta Y_{t}<0\right) P\left(\Delta Y_{t}<0\right)>0
\end{aligned}
$$

for any $A$, which implies that $E[\hat{\xi}]>0$. 


\section{A.2 Proof of Proposition 2}

Proof. The proof will be divided into two parts. We will first derive the equations that define the solution of the Pareto problem. We will then show that the solution of the three-stage formulation satisfies the equations that characterize the solution of the Pareto problem. We will consider only the case in which the group is composed of an even number of households and pairs are formed by taking households 1 and 2,3 and $4, \ldots$, and $n-1$ and $n$. The case of $n$ odd and of a different aggregation of pairs are a straightforward generalization.

Under the assumption that the utility functions are strictly increasing and concave, the first order conditions of the Pareto problem are necessary and sufficient for its solution. For each period $t$ they imply the following set of $2 n$ equations in $2 n$ unknowns:

$$
\begin{array}{rlrl}
\frac{u_{l}^{i}\left(c_{t}^{i}, l_{t}^{i}\right)}{u_{c}^{i}\left(c_{t}^{i}, l_{t}^{i}\right)} & =w_{t}^{i}, & & i=1, \ldots, n, \\
\mu^{i} u_{c}^{i}\left(c_{t}^{i}, l_{t}^{i}\right) & =\mu^{i+1} u_{c}^{i+1}\left(c_{t}^{i+1}, l_{t}^{i+1}\right), & & i=1, \ldots, n-1, \\
\sum_{i=1}^{n}\left(c_{t}^{i}+w_{t}^{i} l_{t}^{i}\right) & =\bar{Y}_{t} . &
\end{array}
$$

where $\bar{Y}_{t}$ is full income for the group. These equations fully characterize the solution of the Pareto problem for each $t$.

We will now show that the equations (12), (13), and (14) are satisfied at the solution of the three-stage problem. As for the Pareto problem, the solution of the three-stage problem is fully characterized by its first order conditions. Let $\lambda_{1 t}, \lambda_{2 t}^{2 i-1,2 i}$, and $\lambda_{3 t}^{i}$ be the multipliers associated with the resource constraints of the first, second, and third stage. The first order conditions for the third stage of the problem can then be written as follows:

$$
\begin{aligned}
u_{l}^{i}\left(c_{t}^{i}, l_{t}^{i}\right) & =w_{t}^{i} \lambda_{3 t}^{i}, & & i=1, \ldots, n, \\
u_{c}^{i}\left(c_{t}^{i}, l_{t}^{i}\right) & =\lambda_{3 t}^{i}, & & i=1, \ldots, n-1, \\
c_{t}^{i}+w_{t}^{i} l_{t}^{i} & =\rho_{t}^{i}, & & i=1, \ldots, n .
\end{aligned}
$$

Equations (15) and (16) imply that $\frac{u_{l}^{i}\left(c_{t}^{i}, l_{t}^{i}\right)}{u_{c}^{i}\left(c_{t}^{i}, l_{t}^{i}\right)}=w_{t}^{i}$ for $i=1, \ldots, n$. Moreover, equations (17) imply that $\sum_{i=1}^{n}\left(c_{t}^{i}+w_{t}^{i} l_{t}^{i}\right)=\bar{Y}_{t}$. Hence, equations (12) and (14) of the Pareto problem are satisfied at the solution the three-stage problem.

The third-stage problem can be used to derived one last useful equation. A straightforward application of the envelope theorem implies that

$$
V_{\rho}^{i}=\lambda_{3 t}^{i}=u_{c}^{i}\left(c_{t}^{i}, l_{t}^{i}\right) \quad i=1, \ldots, n .
$$

The first order conditions of the second-stage problem can be written in the following form:

$$
\mu_{2 i-1} V_{\rho}^{2 i-1}=\lambda_{2 t}^{2 i-1,2 i} \quad \text { and } \quad \mu_{2 i} V_{\rho}^{2 i}=\lambda_{2 t}^{2 i-1,2 i}
$$


They imply that the following equations are satisfied:

$$
\mu_{2 i-1} V_{\rho}^{2 i-1}=\mu_{2 i} V_{\rho}^{2 i} \quad i=2, \ldots, n .
$$

The envelope theorem can be applied to the second-stage problem to show that

$$
V_{\rho}^{2 i-1,2 i}=\lambda_{2 t}^{2 i-1,2 i}=\mu_{2 i-1} V_{\rho}^{2 i-1}=\mu_{2 i} V_{\rho}^{2 i} \quad i=2, \ldots, n .
$$

Finally, the first order conditions of the first-stage problem take the following form:

$$
V_{\rho}^{2 i-1,2 i}=\lambda_{1 t} \quad i=2, \ldots, n,
$$

which imply that

$$
V_{\rho}^{2 i-1,2 i}=V_{\rho}^{2 j-1,2 j} \quad i \neq j .
$$

Using Equations (18), (20), and (21) it can be shown that

$$
\mu^{i} u_{c}^{i}\left(c_{t}^{i}, l_{t}^{i}\right)=\mu^{i+1} u_{c}^{i+1}\left(c_{t}^{i+1}, l_{t}^{i+1}\right) \quad i=1, \ldots, n-1 .
$$

As a consequence, the equations (13) of the Pareto problem are also satisfied.

Since the solution of the three-stage problem solves the first order conditions of the Pareto problem, the result follows.

\section{A.3 Proof of Proposition 3}

Proof. This proposition will be proved by contradiction. Suppose household $i$ and $j$ share risk efficiently and that there exist two income realizations $\rho^{i, j}$ and $\bar{\rho}^{i, j}$ such that

$$
\rho^{i}\left(\rho^{i, j} ; w^{i}, w^{j}\right)>\rho^{j}\left(\rho^{i, j} ; w^{i}, w^{j}\right)
$$

and

$$
\rho^{i}\left(\bar{\rho}^{i, j} ; w^{i}, w^{j}\right)<\rho^{j}\left(\bar{\rho}^{i, j} ; w^{i}, w^{j}\right) .
$$

Assume that household $i$ and $j$ have identical risk preferences. This implies that $V^{i}=V^{j}=V$, where $V^{k}$ is the value function of household $k$ introduced in the three-stage formulation of Section 3 . We will show that under these conditions efficiency is violated.

Without loss of generality assume that $\rho^{i, j}<\bar{\rho}^{i, j}$. Strict concavity of the household utility functions implies that the corresponding value functions are strictly concave in income. ${ }^{28}$ Consequently,

$$
V_{\rho}\left(\rho^{j}\left(\rho^{i, j} ; w^{i}, w^{j}\right)\right)>V_{\rho}\left(\rho^{i}\left(\rho^{i, j} ; w^{i}, w^{j}\right)\right) .
$$

This implies,

$$
\mu_{i} V_{\rho}\left(\rho^{j}\left(\rho^{i, j} ; w^{i}, w^{j}\right)\right)>\mu_{i} V_{\rho}\left(\rho^{i}\left(\rho^{i, j} ; w^{i}, w^{j}\right)\right)=\mu_{j} V_{\rho}\left(\rho^{j}\left(\rho^{i, j} ; w^{i}, w^{j}\right)\right),
$$

\footnotetext{
${ }^{28}$ For a proof see for instance Proposition 3.6 in Kreps (1990).
} 
where the equality follows from efficient risk sharing. Hence, $\mu_{i}>\mu_{j}$.

Now consider the realization $\bar{\rho}^{i, j}$. Strict concavity of $V$ implies that

$$
V_{\rho}\left(\rho^{i}\left(\bar{\rho}^{i, j} ; w^{i}, w^{j}\right)\right)>V_{\rho}\left(\rho^{j}\left(\bar{\rho}^{i, j} ; w^{i}, w^{j}\right)\right)
$$

Consequently,

$$
\mu_{i} V_{\rho}\left(\rho^{i}\left(\bar{\rho}^{i, j} ; w^{i}, w^{j}\right)\right)>\mu_{i} V_{\rho}\left(\rho^{j}\left(\bar{\rho}^{i, j} ; w^{i}, w^{j}\right)\right)>\mu_{j} V_{\rho}\left(\rho^{j}\left(\bar{\rho}^{i, j} ; w^{i}, w^{j}\right)\right)
$$

where the first inequality follows from (22) and the second one from $\mu_{i}>\mu_{j}$. This implies that efficiency is not satisfied and hence that the household utility functions cannot be identical.

\section{A.4 Proof of Proposition 4}

Proof. The proposition will be proved by contradiction. Suppose that households $i$ and $j$ share risk efficiently and assume that the risk-sharing function of household $i$ is decreasing in some interval $I=\left[\rho_{l}, \rho_{u}\right]$. We will show that efficiency cannot be satisfied.

Consider two realizations of aggregate income $\rho^{i, j} \in I$ and $\bar{\rho}^{i, j} \in I$ with $\rho^{i, j}>\bar{\rho}^{i, j}$. Since the risk-sharing function of household $i$ is decreasing in $I$ we have,

$$
\rho^{i}\left(\rho^{i, j} ; w^{i}, w^{j}\right) \leq \rho^{i}\left(\bar{\rho}^{i, j} ; w^{i}, w^{j}\right) .
$$

As a consequence,

$$
\mu^{j} V_{\rho}^{j}\left(\rho^{j}\left(\rho^{i, j} ; w^{i}, w^{j}\right)\right)=\mu^{i} V_{\rho}^{i}\left(\rho^{i}\left(\rho^{i, j} ; w^{i}, w^{j}\right)\right) \geq \mu^{i} V_{\rho}^{i}\left(\rho^{i}\left(\bar{\rho}^{i, j} ; w^{i}, w^{j}\right)\right)=\mu^{j} V_{\rho}^{j}\left(\rho^{j}\left(\bar{\rho}^{i, j} ; w^{i}, w^{j}\right)\right)
$$

where the two equalities follow from efficiency and the inequality follows from the strict concavity of $V^{k}\left(\rho^{k}\right)$. Hence, by strict concavity of $V^{k}\left(\rho^{k}\right)$, we have

$$
\rho^{j}\left(\rho^{i, j} ; w^{i}, w^{j}\right) \leq \rho^{j}\left(\bar{\rho}^{i, j} ; w^{i}, w^{j}\right) .
$$

This implies that

$$
\rho^{i}\left(\rho^{i, j} ; w^{i}, w^{j}\right)+\rho^{j}\left(\rho^{i, j} ; w^{i}, w^{j}\right) \leq \rho^{i}\left(\bar{\rho}^{i, j} ; w^{i}, w^{j}\right)+\rho^{j}\left(\bar{\rho}^{i, j} ; w^{i}, w^{j}\right) \leq \bar{\rho}^{i, j}<\rho^{i, j},
$$

which cannot be the efficient allocation of resources since preferences are strictly increasing.

\section{A.5 Proof of Proposition 5}

Proof. Suppose that preferences are separable between consumption and leisure or that $w^{i}$ and $w^{j}$ are constant. Then,

$$
\begin{aligned}
& \rho^{i}=\rho^{i}\left(\rho^{i, j} ; w^{i}, w^{j}\right)=\hat{\rho}^{i}\left(\rho^{i, j}\right), \\
& \rho^{j}=\rho^{j}\left(\rho^{i, j} ; w^{j}, w^{i}\right)=\hat{\rho}^{j}\left(\rho^{i, j}\right),
\end{aligned}
$$


for some functions $\hat{\rho}^{i}$ and $\hat{\rho}^{j}$.

Under the assumption that $\rho^{i}$ and $\rho^{j}$ are strictly increasing functions of $\rho^{i, j}$, equations (23) and (24) can be solved for $\rho^{i, j}$ and equated to obtain,

$$
\left(\hat{\rho}^{i}\right)^{-1}\left(\rho^{i}\right)=\left(\hat{\rho}^{j}\right)^{-1}\left(\rho^{j}\right),
$$

where $\left(\hat{\rho}^{k}\right)^{-1}$ is the inverse function of $\hat{\rho}^{k}$. Let $g: \mathbb{R}^{+} \rightarrow \mathbb{R}^{+}$be a strictly decreasing function and $\mu_{k}$ a scalar satisfying the following conditions: $0<\mu_{k}<1$, for $k=1, \ldots n$, where $n$ is the number of households in the economy; $\sum_{i=1}^{n} \mu_{k}=1$. Then, equation (25) implies that

$$
\mu_{i} \frac{g\left(\left(\hat{\rho}^{i}\right)^{-1}\left(\rho^{i}\right)\right)}{\mu_{i}}=\mu_{j} \frac{g\left(\left(\hat{\rho}^{j}\right)^{-1}\left(\rho^{j}\right)\right)}{\mu_{j}} .
$$

Let the function $V^{k}\left(\rho^{k}\right)$ be defined as follows:

$$
V^{k}\left(\rho^{k}\right)=\frac{1}{\mu_{k}} \int_{0}^{\rho^{k}} g\left(\left(\hat{\rho}^{i}\right)^{-1}(t)\right) d t
$$

The function $V^{k}$ satisfies the following two properties: $V_{\rho}^{k}=g\left(\left(\hat{\rho}^{k}\right)^{-1}\left(\rho^{k}\right)\right) / \mu_{k}>0$ and $V_{\rho \rho}^{k}=$ $g_{\rho}<0$. The first inequality follows from the second fundamental theorem of calculus and $g>0$. The second inequality follows from $g$ being decreasing and the risk-sharing functions being increasing, which implies that $\left(\hat{\rho}^{k}\right)^{-1}$ is also increasing. Consequently, $V^{k}\left(\rho^{k}\right)$ is a well-defined strictly increasing and concave utility function over $\rho^{k}$.

Under separable preferences $\rho^{k}$ is equal to consumption of household $k$. Under the assumption that real wages are constant, the composite commodity theorem shows that consumption and leisure can be treated as a single good and that only the utility function over the composite good $\rho^{k}=c^{k}+w^{k} l^{k}$ is relevant to describe household behavior. ${ }^{29}$ Hence, by (26) there exist strictly increasing and concave utility functions $V^{i}(\rho)$ and $V^{j}(\rho)$ such that the efficiency condition is satisfied, i.e.:

$$
\mu_{i} V_{\rho}^{i}\left(\rho^{i}\right)=\mu_{j} V_{\rho}^{j}\left(\rho^{j}\right) .
$$

\section{A.6 Proof of Proposition 6}

\section{Proof.}

Consider household $i$ and $j$ and suppose that they have identical preferences but different discount factors. Under these assumptions, the second stage of the three-stage formulation of the Pareto problem must be modified as follows to include the heterogeneous discount factors:

$$
\begin{gathered}
\max _{\rho_{t}^{i}, \rho_{t}^{j}} \mu_{i} \beta_{i}^{t} V\left(\rho_{t}^{i} ; w_{t}^{i}, z_{t}^{i}, \eta_{t}^{i}\right)+\mu_{j} \beta_{j}^{t} V\left(\rho_{t}^{j} ; w_{t}^{j}, z_{t}^{j}, \eta_{t}^{j}\right) \\
\text { s.t. } \rho_{t}^{i}+\rho_{t}^{j}=\rho_{t}^{i, j} .
\end{gathered}
$$

\footnotetext{
${ }^{29}$ See Hicks (1990) for a proof of the composite commodity theorem.
} 
Its first order conditions take the following form:

$$
\frac{V_{\rho}\left(\rho_{t}^{i}\right)}{V_{\rho}\left(\rho_{t}^{j}\right)}=\frac{\mu_{j}}{\mu_{i}}\left(\frac{\beta_{j}}{\beta_{i}}\right)^{t} \quad \text { for each } t, \omega .
$$

Without loss of generality suppose that $\mu_{i}=\mu_{j}$ and let $K=\left(\frac{\beta_{j}}{\beta_{i}}\right)^{t}$. Consider the case in which $\beta_{j}>\beta_{i}$ and hence $K>1$. Given the assumption that the household preferences are strictly increasing, $V_{\rho}$ is positive which implies that

$$
V_{\rho}\left(\rho_{t}^{i}\right)>V_{\rho}\left(\rho_{t}^{j}\right) \text { for each } t, h_{t} .
$$

Household preferences are assumed to be strictly concave, which implies that $V_{\rho}$ is decreasing. As a consequence,

$$
\rho_{t}^{i}<\rho_{t}^{j} \text { for each } t, h_{t},
$$

from which the result follows. Finally, one can use the same steps to show that $\rho_{t}^{i}>\rho_{t}^{j}$ for each $t$ and $h_{t}$ if $\beta_{j}<\beta_{i}$.

\section{B Statistical Appendix}

\section{B.1 Estimation of the Risk-sharing Functions}

We will briefly describe how the estimators proposed by Newey et al. (1999) and Ichimura (1993) can be employed to recover the risk-sharing functions. Suppose first that the parameters defining the heterogeneity term $d^{i, j}$ are known. Observe that the risk-sharing functions cannot be estimated using standard non-parametric methods because $E\left[m^{k} \mid \rho^{i, j}\right] \neq 0$. To address this issue, following Newey et al. (1999) let $q$ be a set of instruments in the sense that the following conditions are satisfied:

$$
\rho^{i, j}=h\left(q^{i, j}\right)+\varphi^{i, j}, \quad E\left[\varphi^{i, j} \mid q^{i, j}\right]=0, \text { and } E\left[m^{k} \mid \varphi^{i, j}, q^{i, j}\right]=E\left[m^{k} \mid \varphi^{i, j}\right] .
$$

Then, we have that

$$
\begin{gathered}
E\left[\ln \rho^{k} \mid \rho^{i, j}, w^{i}, w^{j}, d^{i, j}, q^{i, j}\right]=g^{k}\left(\rho^{i, j}, w^{i}, w^{j}, d^{i, j}\right)+E\left[m^{k} \mid \rho^{i, j}, w^{i}, w^{j}, d^{i, j}, q^{i, j}\right] \\
=g^{k}\left(\rho^{i, j}, w^{i}, w^{j}, d^{i, j}\right)+E\left[m^{k} \mid \varphi^{i, j}, q^{i, j}\right]=g^{k}\left(\rho^{i, j}, w^{i}, w^{j}, d^{i, j}\right)+\lambda\left(\varphi^{i, j}\right),
\end{gathered}
$$

where $\lambda(\varphi)=E\left[m^{k} \mid \varphi\right]$. Newey et al. (1999) propose to estimate the function $g^{k}$ in two steps. In the first step the error term $\varphi$ is estimated non-parametrically as $\hat{\varphi}^{i, j}=\rho^{i, j}-\hat{h}\left(q^{i, j}\right)$. In the second step, the function $g^{k}\left(\rho^{i, j}, w^{i}, w^{j}, d^{i, j}\right)+\lambda\left(\varphi^{i, j}\right)$ is estimated using the estimated residuals in place of the true ones. An estimator of the function $g^{k}$ can then be recovered by isolating the components that do not depend on the residuals $\varphi$. The estimation will be performed using the series estimator proposed by Newey et al. (1999) with polynomials. 
The parameters of the heterogeneity term $d^{i, j}$ are not known, but they can be estimated using one of the semi-parametric methods developed for the estimation of single-index models. We use the semi-parametric least square approach proposed by Ichimura (1993). Specifically, each risk-sharing function is estimated by iterating the following two steps. First, for a given value of the parameters in $d^{i, j}$ we estimate the risk-sharing function using the series estimator proposed by Newey et al. (1999). We then compute the sum of squared errors corresponding to the estimated function. We stop when the sum of squared errors is minimized.

\section{B.2 Test of Homogeneous Risk Preferences For a Pair of Households}

The test is implemented in three steps. In the first step, the difference between risk-sharing functions is estimated using the method discussed in the previous subsection. Let $\hat{g}^{i, j}$ be the estimated difference. In the second step, the test statistic $\hat{\xi}_{1}^{i, j}$ is computed as follows:

$$
\hat{\xi}_{1}^{i, j}=-\left(\sum_{l=1}^{n} \mathbf{1}_{\left\{\hat{g}^{i, j}\left(\rho_{l}^{i, j}\right) \geq 0\right\}} \hat{g}^{i, j}\left(\rho_{l}^{i, j}\right)\right)\left(\sum_{l=1}^{n} \mathbf{1}_{\left\{\hat{g}^{i, j}\left(\rho_{l}^{i, j}\right)<0\right\}} \hat{g}^{i, j}\left(\rho_{l}^{i, j}\right)\right),
$$

where $\mathbf{1}_{\{\}}$is an indicator function. In the final step, the distribution of $\hat{\xi}_{1}^{i, j}$ is recovered by bootstrap. To increase the power of the test, we follow Hall and Wilson (1991) and compute the bootstrap distribution by resampling $\hat{\xi}_{1}^{i, j *}-\hat{\xi}_{1}^{i, j}$ instead of $\hat{\xi}_{1}^{i, j *}$, where $\hat{\xi}_{1}^{i, j *}$ is the estimated test statistic obtained using a bootstrap sample. The null is then rejected for the pair composed of households $i$ and $j$ if $\hat{\xi}_{1}^{i, j}$ is too large, i.e. if

$$
\hat{\xi}_{1}^{i, j}>q^{i, j *}(0.95)
$$

where $q^{i, j *}(0.95)$ is the 95 -th percentile of $\hat{\xi}_{1}^{i, j *}-\hat{\xi}_{1}^{i, j}$.

\section{B.3 Test of Efficiency Based on Omitted Variables for a Pair of Households}

This test is implemented using the semi-parametric approach proposed by Fan and Li (1996). We will briefly describe the method. Consider the pair composed of households $i$ and $j$ and suppose that the risk-sharing function of household $i$ depends on an omitted variable $y^{i}$. Then, Subsection B.1 implies that the function can be written in the following form:

$$
\ln \rho^{i}=g^{i}\left(\rho^{i, j}, w^{i}, w^{j}, d^{i, j}, y^{i}\right)+\lambda\left(\varphi^{i, j}\right)+\epsilon^{i}=f^{i}\left(X^{i, j}, y^{i}\right)+\epsilon^{i},
$$

where $E\left[\epsilon^{i} \mid X^{i, j}, y^{i}\right]=0$. Under the null hypothesis of efficiency, $y^{i}$ should not enter the risk-sharing function. As a consequence

$$
f^{i}\left(X^{i, j}, y^{i}\right)=E\left[\ln \rho^{i} \mid X^{i, j}, y^{i}\right]=E\left[\ln \rho^{i} \mid X^{i, j}\right]=r^{i}\left(X^{i, j}\right) .
$$

But under the alternative hypothesis we have that

$$
f^{i}\left(X^{i, j}, y^{i}\right) \neq r^{i}\left(X^{i, j}\right) .
$$


Let $\nu^{i}=\ln \rho^{i}-r^{i}\left(X^{i, j}\right)$. Then, $E\left[\nu^{i} \mid X^{i, j}, y^{i}\right]=f^{i}\left(X^{i, j}, y^{i}\right)-r^{i}\left(X^{i, j}\right)=0$ under the null and $E\left[\nu^{i} \mid X^{i, j}, y^{i}\right] \neq 0$ under the alternative. Now observe that

$$
E\left[\nu^{i} E\left[\nu^{i} \mid X^{i, j}, y^{i}\right]\right]=E\left[\left\{E\left[\nu^{i} \mid X^{i, j}, y^{i}\right]\right\}^{2}\right] \geq 0
$$

where the inequality follows from the previous discussion and the inequality is replaced by an equality if and only if the null hypothesis is correct. Fan an Li propose to test for the omitted variable $y^{i}$ using this inequality and the following idea. If $\nu^{i}$ and $E\left[\nu^{i} \mid X^{i, j}, y^{i}\right]$ were known, one could estimate $E\left[\nu^{i} E\left[\nu^{i} \mid X^{i, j}, y^{i}\right]\right]$ using its sample analog $n^{-1} \sum_{i} \nu^{i} E\left[\nu^{i} \mid X^{i, j}, y^{i}\right]$. The authors suggest to replace the residuals $\nu^{i}$ with estimated ones and $E\left[\nu^{i} \mid X^{i, j}, y^{i}\right]$ with its kernel estimator. Finally, to overcome the random denominator problem in the kernel estimation, they propose to replace the sample analog with its density weighted version:

$$
\frac{1}{n} \sum_{i}\left[\nu^{i} f\left(X^{i, j}\right)\right] E\left[\nu^{i} f\left(X^{i, j}\right) \mid X^{i, j}, y^{i}\right] f\left(X^{i, j}, y^{i}\right)
$$

where $f($.$) denotes the probability density function. Finally, the estimated sample analog is divided$ by its estimated standard deviation and multiplied by $n h^{d / 2}$, where $n$ is the number of observations and $h$ is a smoothing parameter in the kernel estimator. In the present paper we estimate the residuals using the series estimator described in Subsection B.1 and the densities using a standard gaussian kernel estimator.

At this point we have one test statistic for each household in the pair. To compute the test statistic for the pair observe that efficiency is rejected if $y^{i}$ affects the risk-sharing function of at least one household. We can therefore compute the test statistic for the pair $\hat{\xi}_{2}^{i, j}$ by taking the maximum of the individual test statistics. Similarly to the homogeneity test, the distribution of the test statistic for the pair is obtained using bootstrap by resampling $\hat{\xi}_{2}^{i, j *}-\hat{\xi}_{2}^{i, j}$. The null hypothesis is then rejected for the pair composed of households $i$ and $j$ if $\hat{\xi}_{2}^{i, j}$ is too large, i.e. if

$$
\hat{\xi}_{2}^{i, j}>q^{i, j *}(0.95) \text {. }
$$

\section{B.4 Test of Efficiency Based on Increasing Risk-sharing Functions For a Pair}

We will first provide the intuition underlying the test introduced by Hall and Heckman (2000) using a simpler version of the economy considered in this paper. We will then consider the more general case. Suppose that preferences are separable between consumption and leisure, there is no observable and unobservable heterogeneity, and no measurement errors. In this case, household $i$ 's expenditure is only a function of $\rho^{i, j}$ and there is no endogeneity issue, i.e.

$$
\ln \rho^{i}=g\left(\rho^{i, j}\right)+\epsilon
$$

Let $\left\{\left(\ln \rho_{t}^{i}, \rho_{t}^{i, j}\right), 1 \leq t \leq T\right\}$ be data generated by equations $(27)$ and denote by $\left\{\left(\ln \bar{\rho}_{t}^{i}, \bar{\rho}_{t}^{i, j}\right), 1 \leq t \leq T\right\}$ the same data sorted in increasing order of aggregate resources $\rho^{i, j}$. Consider a subset of the sorted 
data $\left\{\left(\ln \bar{\rho}_{t}^{i}, \bar{\rho}_{t}^{i, j}\right), r \leq t \leq s\right\}$ and estimate the slope of the linear regression of $\ln \rho^{i}$ on $\rho^{i, j}$. Repeat the last step for any subset of the sorted data that contains enough information to estimate the slope. Hall and Heckman's idea is that under the hypothesis that the function $g\left(\rho^{i, j}\right)$ is increasing, the minimum over all the estimated slopes should not be negative.

Formally the test is implemented as follows. For a given integer $m$ that will be defined later, let $r$ and $s$ be integers that satisfy $0 \leq r \leq s-m \leq T-m$ and let $a$ and $b$ be scalars. Denote by $h\left(w^{i}, w^{j}, d^{i, j}\right)$ a polynomial in the wages and heterogeneity term, and by $\delta\left(\varphi^{i}\right)$ a polynomial in the first stage residuals in the estimator proposed by Newey et al. (1999). Define

$$
S(a, b, h, \delta \mid r, s)=\sum_{t=r+1}^{s}\left\{\ln \rho_{t}^{i}-\left[a+b \rho_{t}^{i, j}+h\left(w_{t}^{i}, w_{t}^{j}, d_{t}^{i, j}\right)+\delta\left(\varphi_{t}^{i}\right)\right]\right\}^{2} .
$$

For each choice of $r$ and $s$, let $\hat{a}(r, s), \hat{b}(r, s), \hat{h}(r, s)$, and $\hat{\delta}(r, s)$ be the solution of the following least square problem:

$$
(\hat{a}, \hat{b}, \hat{h}, \hat{\delta})=\operatorname{argmin} S(a, b, h, \delta \mid r, s) .
$$

The variance matrix of the estimated coefficients is equal to $\sigma^{2}\left(X^{\prime} X\right)^{-1}$, where $\sigma^{2}$ is the variance of the residuals in the risk-sharing function and $X$ is the matrix of regressors. This implies that the variance of $\frac{\hat{b}}{\sqrt{\left(X^{\prime} X\right)_{b, b}^{-1}}}$ is equal to $\sigma^{2}$, where $\left(X^{\prime} X\right)_{b, b}^{-1}$ is the diagonal element of the inverse matrix that corresponds to $\hat{b}$. The test statistic for each household in the pair can then be defined as

$$
\hat{\xi}_{3}^{i}=\max \left\{-\frac{\hat{b}(r, s)}{\sqrt{\left(X^{\prime} X\right)_{b, b}^{-1}}}: 0 \leq r \leq s-m \leq T-m\right\} .
$$

Note that the integer $m$ plays the role of a smoothing parameter in the sense that larger values of $m$ reduce the effect of outliers. Similarly to the first efficiency test, the test statistic for the pair $\hat{\xi}_{3}^{i, j}$ can be computed by taking the maximum of the two individual test statistics. The test rejects the null if $\hat{\xi}_{3}^{i, j}$ is too large.

The distribution of the test statistic is derived using the bootstrap method suggested by Hall and Heckman (2000). According to this method, the bootstrap distribution should be derived under the hypothesis that the function under investigation is constant in $\rho^{i, j}$ because it is the most difficult nondecreasing function for which to test. As in the previous tests, the bootstrap distribution is obtained by resampling $\hat{\xi}_{3}^{i, j *}-\hat{\xi}_{3}^{i, j}$. We can then reject the null for the pair composed by households $i$ and $j$ if

$$
\hat{\xi}_{3}^{i, j}>q^{i, j *}(0.95)
$$

\section{B.5 Tests at the Economy Level}

The tests at the economy level are based on the multiple testing procedure developed in Romano, Shaikh, and Wolf (2006). Consider $n$ hypotheses $H_{1}, \ldots, H_{n}$ and let $T_{1}, \ldots, T_{n}$ be the associated test 
statistics. Suppose that one is interested in a null hypothesis $H_{0}$ which is equal to the intersection of $H_{1}, \ldots, H_{n}$, in the sense that $H_{0}$ is not rejected only if each individual hypothesis $H_{k}$ is not rejected. Romano, Shaikh, and Wolf (2006) propose a method for testing $H_{0}$ that controls the $k$-FWE rate, i.e. the probability of rejecting at least $k$ true hypotheses. To describe it, let $T_{r_{1}} \geq T_{r_{2}} \geq \ldots \geq T_{r_{n}}$ be the test statistics ordered from the highest to the lowest. For any subset of individual hypotheses $D$, denote by $k-\max \left\{T_{i}\right\}$ the $k$-th largest test statistic and by $c_{D}(1-\alpha, k)$ the $1-\alpha$ percentile of its sampling distribution. In the first step, all the individual hypotheses are considered, i.e. $D=D_{1}=$ $\left\{H_{r_{1}}, \ldots, H_{r_{n}}\right\}$, and the following generalized confidence region is constructed:

$$
\left[T_{r_{1}}-c_{D_{1}}, \infty\right) \times \ldots \times\left[T_{r_{n}}-c_{D_{1}}, \infty\right)
$$

The individual hypothesis $T_{r_{i}}$ is then rejected if $0 \notin\left[T_{r_{i}}-c_{D_{1}}, \infty\right)$, or equivalently $T_{r_{i}}>c_{D_{1}}$. If in the first step no individual hypothesis is rejected, the null $H_{0}$ is also not rejected and the procedure stops. If at least one hypothesis is rejected $H_{0}$ is also rejected. If the number of rejections is smaller than $k$, the procedure stops. Otherwise, Romano, Shaikh, and Wolf (2006) show that the power is increased by proceeding to the second step. Let $R_{1}$ be the number of individual hypotheses rejected in the first step. In the second step, one considers the individual hypotheses not yet rejected, i.e. $D=D_{2}=\left\{H_{r_{R_{1}+1}}, \ldots, H_{r_{R_{n}}}\right\}$, and construct the corresponding generalized confidence region:

$$
\left[T_{r_{R_{1}+1}}-c_{D_{2}}, \infty\right) \times \ldots \times\left[T_{r_{R_{n}}}-c_{D_{2}}, \infty\right),
$$

where the threshold $c_{D_{2}}$ is constructed using the following method. Construct all the possible subsets that contain the $n-R_{1}$ hypotheses that were not rejected plus $k-1$ of the rejected hypotheses. Denote by $D_{2, i}$ the $i$-th subset. For each subset compute the $1-\alpha$ percentile of the sampling distribution of the $k$-th largest test statistic $c_{D_{2, i}}(1-\alpha, k)$. The threshold $c_{D_{2}}$ is the maximum of all $c_{D_{2, i}}$. The hypothesis $T_{r_{R_{i}}}$ is then rejected if $T_{r_{R_{i}}}>c_{D_{1}}$. If the number of rejected hypotheses is smaller than $k$ one should stop. Otherwise, one continues in this stepwise fashion until less then $k$ hypotheses are rejected. The $1-\alpha$ percentile of the sampling distribution of the $k$-th largest test statistic is computed using the bootstrap method illustrated in Romano, Shaikh, and Wolf (2006).

In the empirical implementation of the tests, $k$ will be set equal to a fraction of the individual hypotheses considered. The fraction is chosen following the results of the simulation study that is discussed in the next appendix. It is important to remark that $k$ will be set equal to the same fraction of hypotheses for every group of households. As a consequence, the $k$-FWE rate will be fixed at the same percentage of hypotheses for groups that are characterized by 20 hypotheses as well for groups that are characterized by 500 hypotheses. This choice implies that the same definition of size of the test will be used for small and large groups of households. The only exceptions are groups for which the constant fraction of hypotheses implies a $k$ that is smaller than 1 . In those situations we will set $k$ equal to 1 and the the test will be more likely to reject. 


\section{Simulation Study}

In this appendix we will study the performance of the three tests proposed in this paper using simulations. The simulation study is important for three reasons. First, the statistics used to test homogeneity in preferences and full-insurance are not smooth functions. This feature may be problematic if one wants to bootstrap their distributions. The study will enable us to evaluate the performance of the tests when the distributions are bootstrapped. Second, under the assumption made in Section 6, the measurement errors enter non-linearly in aggregate resources, which may be problematic for the estimator proposed by Newey et al. (1999). With the simulation study we will be able to understand what is the effect of the measurement errors on the performance of the tests. Finally, the results of the simulations will enable us to evaluate the power and control of the tests and to improve them when the tests are implemented using the ICRISAT data. Following Romano, Shaikh, and Wolf (2006) we will focus on three measures of test performance: the average number of false hypotheses rejected; the average number of true hypotheses rejected; the empirical FWE rate.

All the simulations share the following features. To be consistent with the data, it is assumed that the group under investigation is composed of thirty households. ${ }^{30}$ All households have a utility function which is nonseparable between consumption and leisure and has the following form:

$$
u^{i}(c, l ; z, \eta)=\frac{\left(c^{\sigma_{i}} l^{1-\sigma_{i}}+a_{i}\right)^{1-\gamma_{i}}}{1-\gamma_{i}} \exp \{\theta z+\eta\} .
$$

This utility generates risk-sharing functions that satisfy the restrictions discussed in Section 6 . The parameters $\sigma_{i}$ and $a_{i}$ are assumed to be identical across households with $\sigma_{i}=0.5$ and $a_{i}=1$. The risk aversion parameter $\gamma_{i}$ is allowed to vary across households. Fifteen households are assumed to have $\gamma_{i}=1.2$, whereas the corresponding parameter for the other fifteen is set to 2.5. Households can save using a risk-free asset with no constraint on their borrowing ability. The interest rate is fixed at 0.05 and the discount factor is set equal to 0.95 . Each household can draw a high or low daily wage with equal probability. The high and low wages are set equal to 3 and 5 rupees, respectively. We allow for unobservable heterogeneity in the form of a pair fixed effect and for observable heterogeneity using the following three variables: mean adult age, caste ranking, and number of infants. Household decisions are simulated for 160 periods. To approximate the length of the panel in the ICRISAT, the test is then performed using the 120 periods that are between $t=21$ and $t=140$. The simulation is repeated 500 times. The distribution of the test statistics is determined using 500 bootstraps.

To evaluate the effect of measurement errors on the outcome of the tests, we add measurement errors to the natural logarithm of household expenditure $\rho^{i}$. We consider two types of measurement errors. In the first case, they are drawn from a normal distribution with mean zero and a negligible standard deviation $\left(\sigma_{m}=0.01\right)$. In the second case they are drawn from a normal distribution with the same mean but a standard deviation that is equal to half the standard deviation of the

\footnotetext{
${ }^{30}$ We have also performed the simulation study with 10 households obtaining similar results.
} 
simulated household expenditure. We expect the two standard deviations used in the simulation to be a lower and upper bound for the standard deviation of the measurement errors in the data. ${ }^{31}$ In the homogeneity in risk preferences test, the effect of measurement errors depends also on the quality of the instruments. In the simulation of that test we consider two sets of instruments. The first set contains non-labor income, the demographic variables, the wages of each household in the group, and lagged savings. It generates an average $R^{2}$ for total expenditure of about 0.97 , where the average is computed across pairs. The second set contains the wages of the two households considered in the test, lagged total expenditure, lagged savings, and rain which is an exogenous shock in the simulation. In this case, the average $R^{2}$ is about 0.5 .

The implementation of tests requires the researcher to choose $k$ in the $k$-FWE rate and the order of the polynomial in $\rho^{i j}$. We experimented with several values for $k$. We report the results for $k$ equal to 2.5 percent, 5 percent, and 10 percent of total hypotheses, since generally for $k$ greater than 10 percent the loss in control more than dominates the gain in power. For the first two tests, we also experimented with polynomials of order 1,2, 3, and 5. For each one, we will report only the results that are useful to understand the relationship between the order of the polynomial and the test performance. By construction, the efficiency test based on increasing functions does not depend on the order of the polynomial in $\rho^{i j}$.

In the implement of the homogeneity in risk preferences test one must control for the variation in wages, and in observable and unobservable heterogeneity. We control for this variation in two steps. We first estimate semi-parametrically the risk-sharing functions and their differences. We then fix wages and the heterogeneity term at the household mean and perform the tests.

\section{C.1 Simulations for the Test of Homogeneity in Risk Preferences}

In this subsection we evaluate the performance of the test of homogeneity in risk preferences. To that end, we simulate the decisions of the thirthy households under the maintained assumption of efficient risk sharing. The Pareto weights are chosen so that the risk-sharing functions of households with heterogenous risk preferences cross. The goal of the simulation study is therefore to evaluate whether different specifications of the test can detect these crossings. The actual data may correspond to Pareto weights for which the household risk-sharing functions do not cross even if preferences are heterogeneous. The results should therefore be interpreted as an upper bound for the power of the test.

The results indicate that the performance of the test depends on four features of the simulated data: the severity of the measurement error problem; the quality of the instruments used to address it; the order of the polynomial in total expenditure; the choice of $k$ in the $k$-FWE rate. The results are reported in Table 6 for a polynomial in $\rho^{i j}$ of order 2 , which gives the best results. To understand

\footnotetext{
${ }^{31}$ Ravallion and Chaudhuri (1997) convincingly argue that there are measurement errors in the ICRISAT. We could not find a paper, however, that estimates their standard deviation.
} 
them it is important to remember that the risk aversion parameter is equal to 1.2 for fifteen households and to 2.5 for the remaining fifteen. In the simulation there are therefore 225 pairs with heterogeneous preferences and risk-sharing functions that cross, and 210 pairs with homogeneous risk preferences.

We start the description of the results by discussing the effect of the measurement errors on the outcome of the test. If the standard deviation of the measurement errors is negligible, the test can easily detect the existence of heterogeneity in risk preferences. The average rejection of false hypotheses is 224.5 out of 225 . If the standard deviation is large the average number of false hypotheses rejected by the test drops significantly for some specifications of the test. The lowest average is obtained when we employ the small set of instruments and $k=11$. For this specification the average number of correct rejections is just $23.4 \%$ of the total. The number increases significantly if we use the large set of instruments and we set $k$ equal to 44 . In this case the average number of correct rejections is $80 \%$. To understand why measurement errors have a substantial effect on the power of the test, observe that the test statistic is constructed using the difference in risk-sharing functions. In the simulation the difference is generally much smaller than the actual expenditures. When the measurement errors are drawn from a distribution with a standard deviation that equals half the standard deviation of household expenditure, the difference in measurement errors dominates the difference in true expenditures in many instances. It is therefore difficult for the test to detect a crossing unless a strong set of instruments is used and $k$ is increased. The measurement errors have very little effect on the number of true hypotheses rejected by the test. The average number of false rejections is always very small with the highest number being $10 \%$ of the total for the large set of instruments, high $\sigma_{m}, k=44$.

The simulation study also indicates that the choice of $k$ has an important effect on the power of the test. When the standard deviation of the measurement errors is large the test is too conservative if $k$ is set equal to 11 . When we increase $k$ to 22 or 44 we observe a substantial gain in power with little loss in control. For instance when we use the large set of instruments, an increase in $k$ from 11 to 22 raises the average number of correct rejections from $63 \%$ to $72.2 \%$ of total rejections. At the same time the average number of false rejections increases only slightly from $0.5 \%$ to $2.5 \%$. An additional increase in $k$ to 44 increases the power of the test with $80 \%$ of false hypotheses rejected. But at same time, the number of true hypotheses rejected becomes four times as large. When we use the small set of instruments, $k=44$ appears to be the optimal choice.

The test of homogeneity in risk preferences in the ICRISAT will be set up taking into account the results of the simulation study. We expect the standard deviation of the measurement errors to be between the ones considered in the simulation study. Moreover, the set of instruments that will be used produces an average $R^{2}$ of about 0.71 . Because of all this, we will implement the test using the following specification. We will set $k$ equal to $5 \%$ of the total number of individual hypotheses and the order of the polynomial to 2 . 


\section{C.2 Simulations for the Efficiency Test Based on Non-labor Income}

In this subsection we will discuss the performance of the efficiency test based on non-labor income. Its evaluation requires the simulation of an economy in which a first group of households share risk efficiently, whereas efficiency is violated for a second group. We will assume that fifteen households behave efficiently and that the remaining fifteen are in autarky and can insure themselves against income shocks only by using the risk-free asset. We therefore have 435 individual hypotheses, 330 of which are false. It is important to point out that autarky with a risk-free asset is only one possible alternative to efficiency. Other alternatives are autarky without savings, cooperation without commitment, and cooperation with asymmetric information. We have chosen autarky with a risk-free asset because it has been shown in the finance literature that in this environment households can achieve a degree of insurance similar to the degree that can be achieved in an economy with efficient risk sharing.

The ability of households in autarky to insure themselves against income shocks using the risk-free asset depends on the properties of the non-labor income process. In the simulation we consider a process that attempts to replicate non-labor income in the ICRISAT data. It is assumed that the process is distributed according to a normal distribution with a mean that depends on lagged nonlabor income, mean adult age, caste, and number of infants. This specification enables us to capture the fact that non-labor income is highly persistent in the data: everything else equal, an increase in lagged non-labor income by $100 \%$ increases current non-labor income by about $50 \%$. Using this specification and the ICRISAT data we can estimate the mean and variance of the process. We can then compute the probability of drawing different realizations for non-labor income.

The simulation results for the first efficiency test differ from the results obtained from the homogeneity test in two respects. First, in the efficiency test the measurement errors have a smaller effect on the outcome of the test. To provide the intuition behind this result note that the present test is based on the estimated risk-sharing functions and not on their differences. Consequently, measurement errors drawn from the same distribution have a smaller effect on the test. For this reason we only report the outcome of the simulation study for the large set of instruments. The second difference is that the best performance is obtained with a polynomial in $\rho^{i, j}$ of order 4 . To understand why this test requires a polynomial of higher order, observe that households that share risk efficiently have risk-sharing functions that are approximately concave in total expenditure after one takes the natural logarithm of household expenditure. A polynomial of order 2 is therefore the optimal choice for this group. The group of inefficient households, however, have risk-sharing functions with a more complicated functional form. As a consequence they require a polynomial of higher order. A polynomial of order 4 enables us to approximate in the best possible way the risk-sharing functions of both groups of households. We only report the results for this specification.

Table 7 describes the outcome of the simulation study. If the standard deviation of the mea- 
surement errors is negligible, the test is able to reject almost all false hypotheses. When $k=11$ we reject on average 328.5 false hypotheses out of 330 . When $k$ is set equal to 22 or 44 we reject all false hypotheses. When we increase the standard deviation, the average number of correct rejections decreases but only slightly with 314.1 average rejections for $k=11,328.7$ rejections for $k=22$, and 329.8 rejections for $k=44$. The average number of true hypotheses rejected is small for both specifications of the measurement errors.

The results of the simulation study are used to set up the test that will be used to evaluate efficiency in Indian villages. The test will be implemented using a polynomial of order 4 in $\rho^{i j}$ and a $k$ that corresponds to $5 \%$ of total hypotheses.

\section{C.3 Simulations for the Efficiency Test Based on Increasing Functions}

In this section we will describe the performance of the efficiency test based on increasing functions. Similarly to the non-labor income test, we simulate an economy in which fifteen households share risk efficiently and fifteen households are in autarky.

The computation of the test statistics for the present test requires a choice for the smoothing parameter $m$. We have experimented with $m=10$ and $m=15$. When the measurement errors have a negligible standard deviation the power of the test is maximized without sacrificing control when $m=10$. If we increase this parameter to 15 , however, the test becomes to conservative and we reject too few false hypotheses. When the measurement errors have a large standard deviation, we reject too many true hypotheses if $m$ is set 10 because the impact of the outlying data is too large. With the high standard deviation we obtain the best balance between power and control when we set $m=15$. In the first part of Table 8 , we report the results for $m=10$ for the low variance case and for $m=15$ for the high variance case.

As mentioned above, by construction the performance of the test is not affected by the choice of the order of the polynomial, but it is by the choice of $k$ for the $k$-FWE rate. We report our findings in Table 8 for the two specifications of the measurement errors and for $k$ equal to 11,22 , and 44 . The test has good power and control when the variance of the measurement errors is low. Its performance is not as good when the variance of the measurement errors is large. In this case, it is difficult for the test to detect the non-monotonicity that characterizes the false hypotheses if $k$ is low. When $k$ is large there is loss of control with the rejection of too many true hypotheses. The choice of $k=22$ appears to achieve the best balanced between power and control.

In the second part of Table 8, we describe the effect of changing the smoothing parameter $m$. Specifically, we fix the standard deviation of the measurement errors to the lower level and $k$ to 22 and then we vary $m$ from 8 to 12 . The results show that an increase in the smoothing parameter reduces the power of the test by decreasing the number of false hypotheses that are rejected, but it has the beneficial effect of of reducing the probability of a type I errors by decreasing the number of 
true hypotheses rejected by the test.

Given the results obtained with the simulations, we will test efficiency based on increasing functions in the ICRISAT by setting the smoothing parameter to 10 and $k$ equal to $5 \%$ of total individual hypotheses.

\section{B Tables}

Table 1: Summary Statistics.

\begin{tabular}{|c|c|c|c|c|c|c|}
\hline \multirow[b]{2}{*}{ Variable } & \multicolumn{2}{|c|}{ Aurepalle } & \multicolumn{2}{|c|}{ Shirapur } & \multicolumn{2}{|c|}{ Kanzara } \\
\hline & Mean & Std. Dev. & Mean & Std. Dev. & Mean & Std. Dev. \\
\hline Grain & 10.9 & 12.8 & 18.5 & 12.2 & 15.1 & 16.5 \\
\hline Food (minus Grain) & 10.4 & 29.4 & 14.2 & 15.5 & 17.3 & 11.5 \\
\hline Non-Durable (minus Grain, Food) & 1.0 & 1.4 & 1.1 & 1.7 & 1.5 & 3.5 \\
\hline Household size & 7.9 & 3.1 & 7.3 & 3.0 & 7.5 & 3.6 \\
\hline Number of Infants & 0.14 & 0.35 & 0.08 & 0.28 & 0.14 & 0.36 \\
\hline Mean Adult age & 38.3 & 6.6 & 39.2 & 6.8 & 37.2 & 7.2 \\
\hline Age-Gender Weight & 6.5 & 2.8 & 6.0 & 2.5 & 6.0 & 2.9 \\
\hline Daily Wage & 2.4 & 2.7 & 3.0 & 2.7 & 3.3 & 2.8 \\
\hline Daily Labor Supply & 5.0 & 3.3 & 6.0 & 4.9 & 6.0 & 4.2 \\
\hline Non-labor Income & 44.0 & 123.6 & 73.7 & 381.1 & 55.8 & 189.9 \\
\hline N. of Households & & 31 & & 31 & & 35 \\
\hline
\end{tabular}

Note: The sample period corresponds to 1975-1985 for Aurepalle and to 1975-1984 for Shirapur and Kanzara. 
Table 2: Test Of Homogeneity In Risk Preferences.

\begin{tabular}{|c|c|c|}
\hline & "N. of Hypotheses/Pairs & N N. of Rejections \\
\hline \multicolumn{3}{|l|}{ Aurepalle } \\
\hline All Households & 443 & $143 / 443$ \\
\hline \multicolumn{3}{|l|}{ By caste } \\
\hline Caste Score $=7.5$ & 10 & $1 / 10$ \\
\hline Caste Score $=18.75$ & 1 & $1 / 1$ \\
\hline Caste Score $=30$ & 10 & $0 / 10$ \\
\hline Caste Score $=55$ & 28 & $1 / 28$ \\
\hline Caste Score $=86.25$ & 10 & $0 / 10$ \\
\hline Caste Score $=97.5$ & 1 & $0 / 1$ \\
\hline \multicolumn{3}{|l|}{ Shirapur } \\
\hline All Households & 410 & $103 / 410$ \\
\hline \multicolumn{3}{|l|}{ By caste } \\
\hline Caste Score $=5$ & 6 & $0 / 6$ \\
\hline Caste Score $=23.75$ & 25 & $1 / 25$ \\
\hline Caste Score $=72.5$ & 85 & $11 / 85$ \\
\hline \multicolumn{3}{|l|}{ Kanzara } \\
\hline All Households & 554 & $139 / 554$ \\
\hline \multicolumn{3}{|l|}{ By caste } \\
\hline Caste Score $=11.25$ & 13 & $0 / 13$ \\
\hline Caste Score $=55$ & 78 & $4 / 78$ \\
\hline Caste Score $=76.25$ & 3 & $1 / 3$ \\
\hline Caste Score $=91.25$ & 19 & $2 / 19$ \\
\hline
\end{tabular}

Note: The sample period corresponds to 1975-1985 for Aurepalle and to 1975-1984 for Shirapur and Kanzara. The order of the polynomial in $\rho^{i, j}$ is 2 . The results are obtained using a $k$ in the k-FWE rate that corresponds to $5 \%$ of total hypotheses. The significance level is 0.05 . Household expenditure is the sum of expenditure on non-durable consumption and expenditure on leisure. The caste ranking corresponds to the one considered in Behrman (1988). 
Table 3: Standard Test of Efficient Risk Sharing

\begin{tabular}{|c|c|c|c|}
\hline & Coefficient on Non-labor Income & Standard Errors & N. Observations \\
\hline \multicolumn{4}{|l|}{ Aurepalle } \\
\hline All Households & $0.261^{* *}$ & 0.018 & 3635 \\
\hline \multicolumn{4}{|l|}{ By caste } \\
\hline Caste Score $=7.5$ & $0.191^{* *}$ & 0.043 & 589 \\
\hline Caste Score $=18.75$ & $0.447^{* *}$ & 0.105 & 238 \\
\hline Caste Score $=30$ & $0.190^{* *}$ & 0.030 & 595 \\
\hline Caste Score $=55$ & $0.258^{* *}$ & 0.039 & 944 \\
\hline Caste Score $=86.25$ & $0.197^{* *}$ & 0.029 & 595 \\
\hline Caste Score $=97.5$ & $0.264^{* *}$ & 0.041 & 238 \\
\hline \multicolumn{4}{|l|}{ Shirapur } \\
\hline All Households & $0.207^{* *}$ & 0.026 & 3242 \\
\hline \multicolumn{4}{|l|}{ By caste } \\
\hline Caste Score $=5$ & $0.124^{* *}$ & 0.054 & 423 \\
\hline Caste Score $=23.75$ & $0.280^{* *}$ & 0.046 & 819 \\
\hline Caste Score $=72.5$ & $0.184^{* *}$ & 0.040 & 1574 \\
\hline \multicolumn{4}{|l|}{ Kanzara } \\
\hline All Households & $0.304^{* *}$ & 0.023 & 3677 \\
\hline Caste Score $=11.25$ & $0.336^{* *}$ & 0.065 & 611 \\
\hline Caste Score $=55$ & $0.263^{* *}$ & 0.039 & 1376 \\
\hline Caste Score $=76.25$ & $0.381^{* *}$ & 0.069 & 321 \\
\hline Caste Score $=91.25$ & $0.324^{* *}$ & 0.042 & 738 \\
\hline
\end{tabular}

See note in Table 2. $\left({ }^{* *}\right)$ and $(*)$ indicate that the coefficient is significant, respectively, at the 5 and 10 percent level. 
Table 4: Tests of Efficient Risk Sharing.

\begin{tabular}{|c|c|c|c|}
\hline & I N. of Hypotheses/Pairs & $\begin{array}{c}\text { N. of Rejections } \\
\text { Increasing Functions }\end{array}$ & $\begin{array}{l}\text { N. of Rejections } \\
\text { Non-labor Income }\end{array}$ \\
\hline \multicolumn{4}{|l|}{ Aurepalle } \\
\hline All Households & 443 & $3 / 443$ & $2 / 443$ \\
\hline \multicolumn{4}{|l|}{ By caste } \\
\hline Caste Score $=7.5$ & 10 & $0 / 10$ & $0 / 10$ \\
\hline Caste Score $=18.75$ & 1 & $0 / 1$ & $0 / 1$ \\
\hline Caste Score $=30$ & 10 & $0 / 10$ & $0 / 10$ \\
\hline Caste Score $=55$ & 28 & $0 / 28$ & $0 / 28$ \\
\hline Caste Score $=86.25$ & 10 & $0 / 10$ & $0 / 10$ \\
\hline Caste Score $=97.5$ & 1 & $0 / 1$ & $0 / 1$ \\
\hline \multicolumn{4}{|l|}{ Shirapur } \\
\hline All Households & 410 & $9 / 410$ & $6 / 410$ \\
\hline \multicolumn{4}{|l|}{ By caste } \\
\hline Caste Score $=5$ & 6 & $0 / 6$ & $0 / 6$ \\
\hline Caste Score $=23.75$ & 25 & $0 / 25$ & $0 / 25$ \\
\hline Caste Score $=72.5$ & 85 & $0 / 85$ & $0 / 85$ \\
\hline \multicolumn{4}{|l|}{ Kanzara } \\
\hline All Households & 554 & $6 / 554$ & $2 / 554$ \\
\hline \multicolumn{4}{|l|}{ By caste } \\
\hline Caste Score $=11.25$ & 13 & $0 / 13$ & $0 / 13$ \\
\hline Caste Score $=55$ & 78 & $1 / 78$ & $1 / 78$ \\
\hline Caste Score $=76.25$ & 3 & $0 / 3$ & $0 / 3$ \\
\hline Caste Score $=91.25$ & 19 & $0 / 19$ & $0 / 19$ \\
\hline
\end{tabular}

See note in Table 2. The order of the polynomial in $\rho^{i, j}$ is 4 for the test based on non-labor income. The outcome of the test based on increasing functions is independent of the polynomial in $\rho^{i, j}$. In the test based on increasing functions, the smoothing parameter $m$ is set to 10 . The $k$ in the $\mathrm{k}-\mathrm{FWE}$ rate is set to $5 \%$ of total hypotheses in both tests. 
Table 5: Average Real Per-capita Transfers and Loans in Rural Villages.

\begin{tabular}{|c|c|c|c|c|c|c|}
\hline \multirow[b]{2}{*}{ Variable } & \multicolumn{2}{|c|}{ Aurepalle } & \multicolumn{2}{|c|}{ Shirapur } & \multicolumn{2}{|c|}{ Kanzara } \\
\hline & Mean & $\%$ of Expen. & Mean & $\%$ of Expen. & Mean & $\%$ of Expe \\
\hline Real Per-capita Non-durable Expen. & 22.3 & - & 33.8 & - & 33.9 & - \\
\hline \multicolumn{7}{|l|}{ Real Per-capita Transfers Given } \\
\hline Total Transfers & 6.3 & $28.3 \%$ & 5.4 & $16.0 \%$ & 3.0 & $8.9 \%$ \\
\hline Total Transfers within Village & 3.0 & $13.5 \%$ & 1.8 & $5.3 \%$ & 1.9 & $5.6 \%$ \\
\hline Tr. to Relatives/Caste Fellows & 5.5 & $24.7 \%$ & 4.7 & $13.9 \%$ & 2.7 & $8.0 \%$ \\
\hline Tr. to Relat./Caste Fellows w. Village & 2.9 & $13.0 \%$ & 1.3 & $3.9 \%$ & 1.8 & $5.3 \%$ \\
\hline \multicolumn{7}{|l|}{ Real Per-capita Transfers Received } \\
\hline Total Transfers & 4.7 & $21.1 \%$ & 7.1 & $21.0 \%$ & 5.4 & $15.9 \%$ \\
\hline Total Transfers within Village & 1.4 & $6.3 \%$ & 2.1 & $6.2 \%$ & 4.6 & $13.6 \%$ \\
\hline Tr. to Relatives/Caste Fellows & 3.2 & $14.4 \%$ & 5.5 & $16.3 \%$ & 3.5 & $10.3 \%$ \\
\hline Tr. to Relat./Caste Fellows w. Village & 0.5 & $2.2 \%$ & 1.1 & $3.3 \%$ & 3.3 & $9.8 \%$ \\
\hline \multicolumn{7}{|l|}{ Real Per-capita Loans } \\
\hline Loans Given & 1.4 & $6.3 \%$ & 4.0 & $11.8 \%$ & 2.5 & $7.4 \%$ \\
\hline Loans Given to Relatives/Caste Fellows & 0.0 & $0 \%$ & 0.3 & $0.9 \%$ & 0.04 & $0.1 \%$ \\
\hline Loans Received & 17.9 & $80.3 \%$ & 20.5 & $60.7 \%$ & 9.3 & $28.4 \%$ \\
\hline Loans Received from Relat./Caste Fellows & 0.3 & $1.4 \%$ & 0.6 & $1.8 \%$ & 0.2 & $0.6 \%$ \\
\hline \multicolumn{7}{|c|}{ Real Per-capita Loans Conditional on a Positive Amount } \\
\hline Loans Given & 32.4 & - & 69.5 & - & 68.0 & - \\
\hline Loans given to Relatives/Caste Fellows & 0.0 & - & 49.0 & - & 28.2 & - \\
\hline Loans Received & 41.3 & - & 59.2 & - & 52.2 & - \\
\hline Loans Received from Relat./Caste Fellows & 40.0 & - & 18.4 & - & 19.5 & - \\
\hline \multicolumn{7}{|l|}{ Exchanged Laborers and Bullocks } \\
\hline Exchanged Laborers & 0.1 & $0.5 \%$ & 0.4 & $1.2 \%$ & 0.1 & $0.3 \%$ \\
\hline Exchanged Bullocks & 0.2 & $0.9 \%$ & 0.5 & $1.5 \%$ & 0.2 & $0.6 \%$ \\
\hline
\end{tabular}

Note: In the ICRISAT questionnaire, a transfer is defined as a transaction in which resources or money change ownership without compensation. A loan is a similar transaction with a compensation. 
Table 6: Simulation Results for the Test of Homogeneity in Risk Preferences

\begin{tabular}{lccc}
\hline Null Hypothesis & \multicolumn{2}{c}{ Homogeneity in Risk Preferences } \\
\hline \hline & $\begin{array}{c}\text { Average N. of False } \\
\text { Hypotheses Rejected }\end{array}$ & $\begin{array}{c}\text { Average N. of True } \\
\text { Hypotheses Rejected }\end{array}$ & $\begin{array}{c}\text { Empirical } \\
\text { FWE Rate }\end{array}$ \\
\hline Large Instrument Set & & & \\
\hline$\sigma_{m}=0.0, \mathrm{k}=11$ & $224.4 / 225(99.7 \%)$ & $1.8 / 210(0.9 \%)$ & 0.0 \\
$\sigma_{m}=0.5 * \sigma_{\text {exp }}, \mathrm{k}=11$ & $142.0 / 225(63.0 \%)$ & $1.1 / 210(0.5 \%)$ & 0.01 \\
\hline$\sigma_{m}=0.0, \mathrm{k}=22$ & $224.5 / 225(99.8 \%)$ & $5.8 / 210(2.8 \%)$ & 0.0 \\
$\sigma_{m}=0.5 * \sigma_{\text {exp }}, \mathrm{k}=22$ & $162.6 / 225(72.2 \%)$ & $5.3 / 210(2.5 \%)$ & 0.01 \\
\hline$\sigma_{m}=0.0, \mathrm{k}=44$ & $224.5 / 225(99.8 \%)$ & $15.2 / 210(7.2 \%)$ & 0.0 \\
$\sigma_{m}=0.5 * \sigma_{\text {exp }}, \mathrm{k}=44$ & $180.1 / 225(80.0 \%)$ & $20.8 / 210(9.9 \%)$ & 0.01 \\
\hline Small Instrument Set & & & 0.0 \\
\hline$\sigma_{m}=0.0, \mathrm{k}=11$ & $216.3 / 225(96.0 \%)$ & $0.38 / 210(0.2 \%)$ & 0.00 \\
$\sigma_{m}=0.5 * \sigma_{\text {exp }}, \mathrm{k}=11$ & $52.6 / 225(23.4 \%)$ & $4.4 / 210(2.1 \%)$ & 0.0 \\
\hline$\sigma_{m}=0.0, \mathrm{k}=22$ & $217.5 / 225(96.7 \%)$ & $2.5 / 210(1.2 \%)$ & 0.0 \\
$\sigma_{m}=0.5 * \sigma_{\text {exp }}, \mathrm{k}=22$ & $85.3 / 225(37.9 \%)$ & $14.2 / 210(6.8 \%)$ & 0.0 \\
$\sigma_{m}=0.0, \mathrm{k}=44$ & $219.1 / 225(97.4 \%)$ & $12.3 / 210(5.8 \%)$ & 0.0 \\
$\sigma_{m}=0.5 * \sigma_{\text {exp }}, \mathrm{k}=44$ & $115.7 / 225(51.4 \%)$ & & \\
\hline
\end{tabular}

Note: The results are obtained by simulating an economy with thirty households that share risk efficiently. Fifteen households have a coefficient of risk aversion equal to 1.2 and fifteen households have a coefficient of risk aversion equal to 2.5. There are therefore 210 pairs for which the null of identical risk preferences is satisfied and 225 pairs for which the null is violated. 
Table 7: Simulation Results for the Test of Efficiency Based on Non-labor Income

\begin{tabular}{lccc}
\hline Null Hypothesis & \multicolumn{2}{c}{ Efficiency Based on Non-labor Income } \\
\hline \hline & $\begin{array}{c}\text { Average N. of False } \\
\text { Hypotheses Rejected }\end{array}$ & $\begin{array}{c}\text { Average N. of True } \\
\text { Hypotheses Rejected }\end{array}$ & $\begin{array}{c}\text { Empirical } \\
\text { FWE Rate }\end{array}$ \\
\hline \multicolumn{1}{c}{ Order of Polynomial in $\rho^{i j}=4$} & $0.4 / 105.0(0.4 \%)$ & 0.0 \\
\hline$\sigma_{m}=0.01, \mathrm{k}=11$ & $328.5 / 330.0(99.4 \%)$ & $1.8 / 105.0(1.7 \%)$ & 0.0 \\
$\sigma_{m}=0.5 * \sigma_{\text {exp }}, \mathrm{k}=11$ & $314.1 / 330.0(95.2 \%)$ & $1.7 / 105.0(1.7 \%)$ & 0.0 \\
$\sigma_{m}=0.01, \mathrm{k}=22$ & $329.6 / 330.0(99.9 \%)$ & $1.7 / 105.0(1.7 \%)$ & 0.0 \\
$\sigma_{m}=0.5 * \sigma_{\exp }, \mathrm{k}=22$ & $328.7 / 330.0(99.6 \%)$ & $9.7 / 105.0(9.0 \%)$ & 0.0 \\
$\sigma_{m}=0.01, \mathrm{k}=44$ & $329.9 / 330.0(100.0 \%)$ & $5.6 / 105.0(5.0 \%)$ & 0.0 \\
$\sigma_{m}=0.5 * \sigma_{\exp }, \mathrm{k}=44$ & $329.8 / 330.0(99.9 \%)$ & & 0 \\
\hline
\end{tabular}

Note: The results are obtained by simulating an economy with thirty households. Fifteen of them share risk efficiently, whereas the remaining fifteen are in autarky. There are therefore 105 pairs for which the null of efficiency is satisfied and 330 pairs for which the null is violated.

Table 8: Simulation Results for the Test of Efficiency Based on Increasing Risk-sharing Functions

\begin{tabular}{lccc}
\hline Null Hypothesis & \multicolumn{2}{c}{ Efficiency Based on Increasing Functions } \\
\hline \hline & $\begin{array}{c}\text { Average N. of False } \\
\text { Hypotheses Rejected }\end{array}$ & $\begin{array}{c}\text { Average N. of True } \\
\text { Hypotheses Rejected }\end{array}$ & $\begin{array}{c}\text { Empirical } \\
\text { FWE Rate }\end{array}$ \\
\hline \hline$\sigma_{m}=0.01, \mathrm{k}=11, \mathrm{~m}=10$ & $265.8 / 330.0(80.6 \%)$ & $1.7 / 105.0(1.6 \%)$ & 0.01 \\
$\sigma_{m}=0.5 * \sigma_{\text {exp }}, \mathrm{k}=11, \mathrm{~m}=15$ & $147.0 / 330.0(44.5 \%)$ & $0.04 / 105.0(0.0 \%)$ & 0.0 \\
\hline$\sigma_{m}=0.01, \mathrm{k}=22, \mathrm{~m}=10$ & $307.1 / 330.0(93.1 \%)$ & $2.0 / 105.0(1.9 \%)$ & 0.0 \\
$\sigma_{m}=0.5 * \sigma_{\text {exp }}, \mathrm{k}=22, \mathrm{~m}=15$ & $273.4 / 330.0(82.9 \%)$ & $2.8 / 105.0(2.6 \%)$ & 0.02 \\
\hline$\sigma_{m}=0.01, \mathrm{k}=44, \mathrm{~m}=10$ & $319.5 / 330.0(96.8 \%)$ & $2.2 / 105.0(2.1 \%)$ & 0.0 \\
$\sigma_{m}=0.5 * \sigma_{\text {exp }}, \mathrm{k}=44, \mathrm{~m}=15$ & $316.1 / 330.0(95.8 \%)$ & $19.8 / 105.0(18.9 \%)$ & 0.09 \\
\hline \hline
\end{tabular}

An Illustration of the Effect of Changing the Smoothing Parameter $m$

\begin{tabular}{llll}
\hline$\sigma_{m}=0.01, \mathrm{k}=22, \mathrm{~m}=8$ & $313.1 / 330.0(94.9 \%)$ & $16.2 / 105.0(15.4 \%)$ & 0.20 \\
\hline$\sigma_{m}=0.01, \mathrm{k}=22, \mathrm{~m}=9$ & $311.1 / 330.0(94.3 \%)$ & $7.9 / 105.0(7.6 \%)$ & 0.03 \\
\hline$\sigma_{m}=0.01, \mathrm{k}=22, \mathrm{~m}=10$ & $307.1 / 330.0(93.1 \%)$ & $2.0 / 105.0(1.9 \%)$ & 0.0 \\
\hline$\sigma_{m}=0.01, \mathrm{k}=22, \mathrm{~m}=11$ & $299.6 / 330.0(89.5 \%)$ & $1.8 / 105.0(1.7 \%)$ & 0.0 \\
\hline$\sigma_{m}=0.01, \mathrm{k}=22, \mathrm{~m}=12$ & $281.3 / 330.0(85.3 \%)$ & $0.2 / 105.0(0.2 \%)$ & 0.0 \\
\hline \hline
\end{tabular}

See note in Table 7 . 


\section{F Figures}

Figure 1: Efficiency Condition with Heterogeneous HARA Preferences and $\mu_{1}=\mu_{2}$.

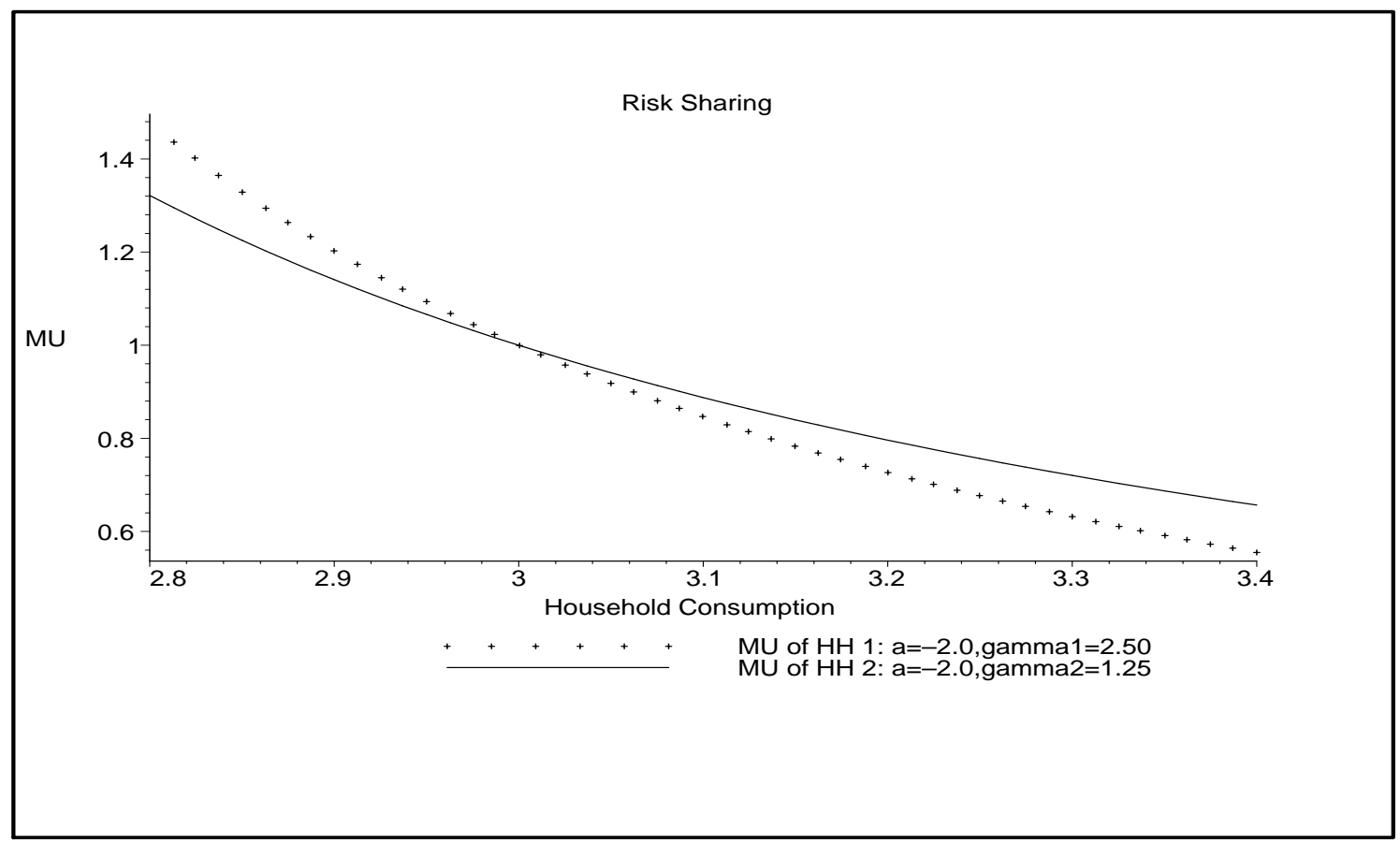

Figure 2: Efficient Consumption with Heterogeneous HARA Preferences and $\mu_{1}=\mu_{2}$.

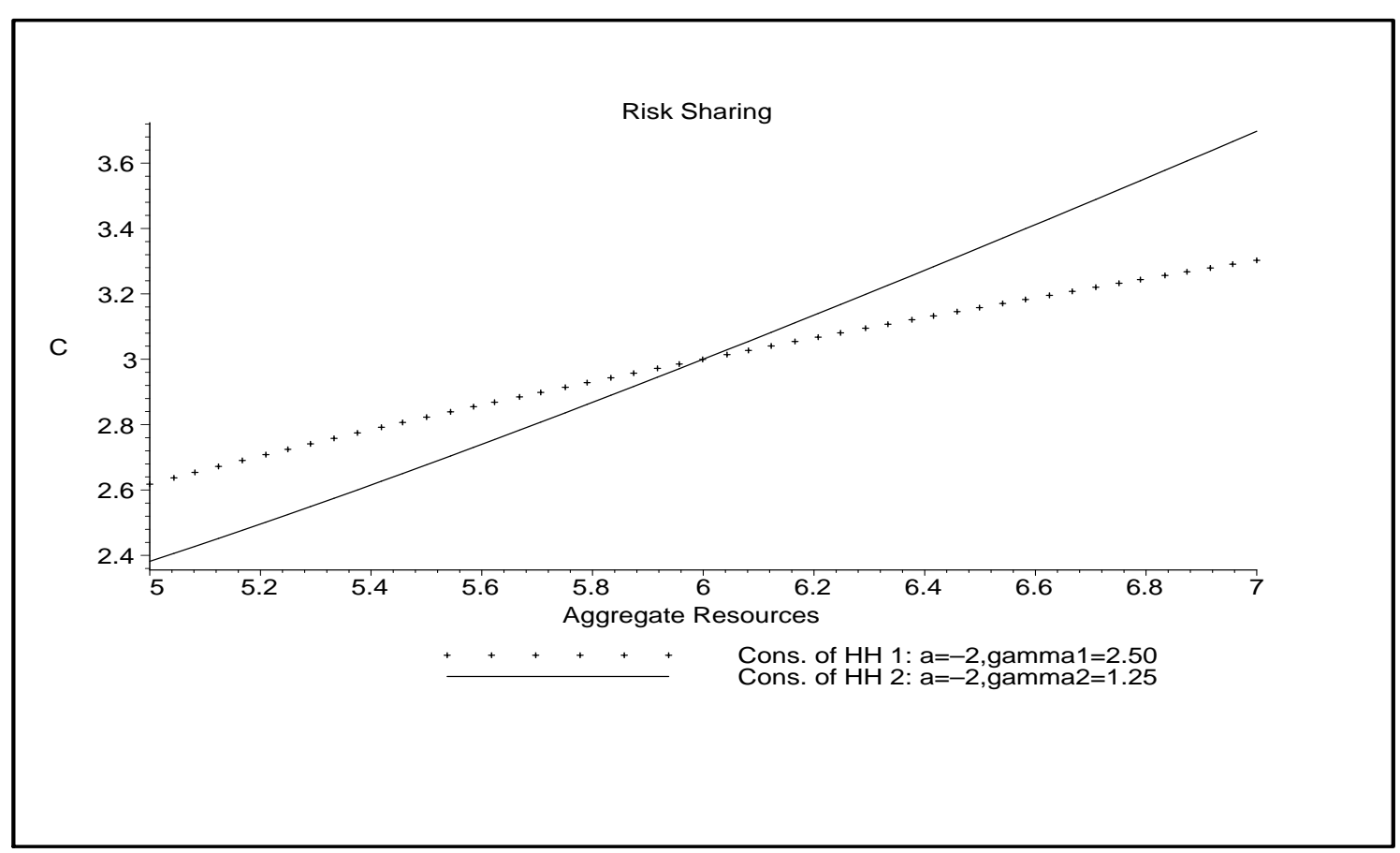


Figure 3: Efficiency Condition with Identical HARA Preferences and $\mu_{1}>\mu_{2}$.

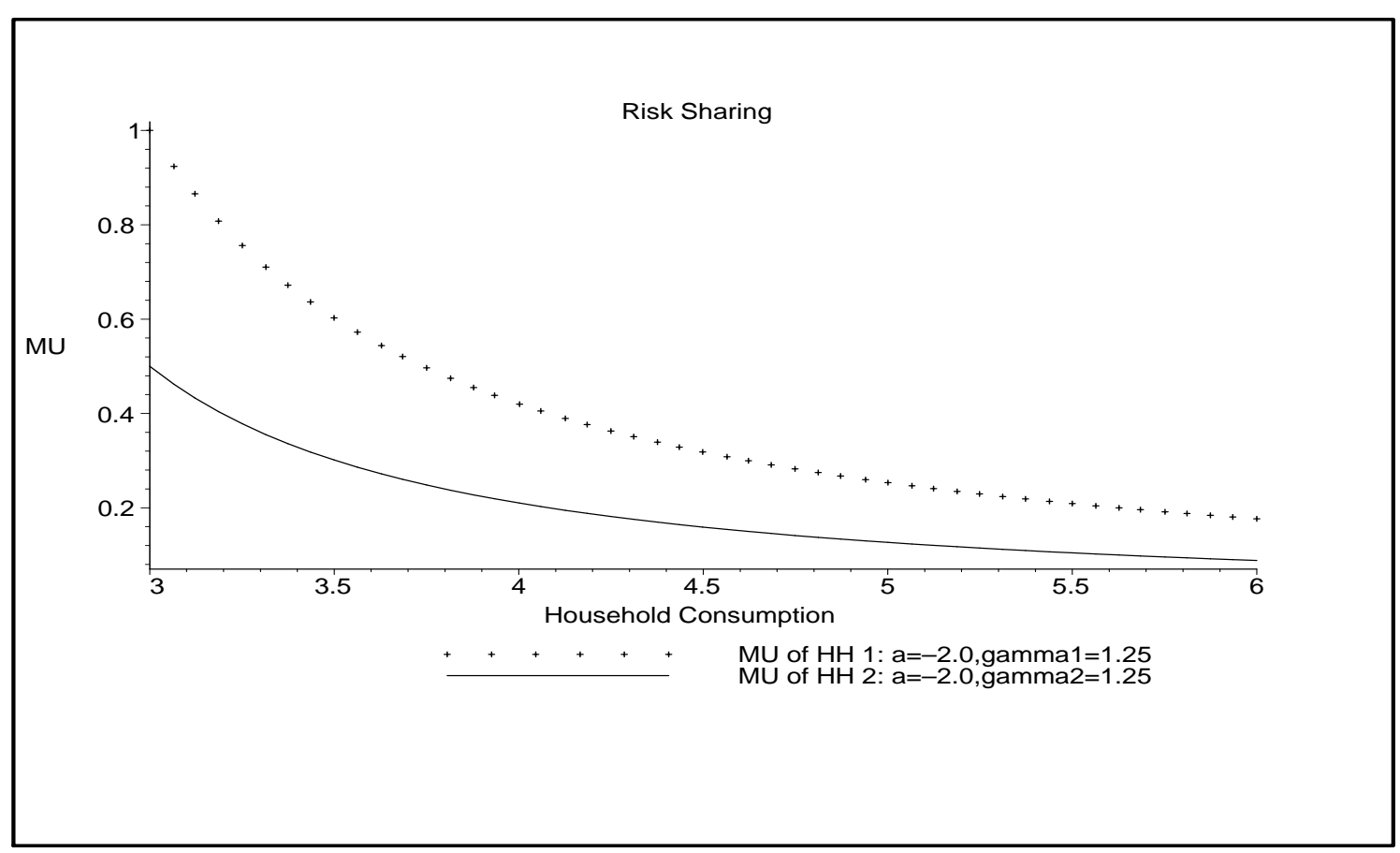

Figure 4: Efficient Consumption with Identical HARA Preferences and $\mu_{1}>\mu_{2}$.

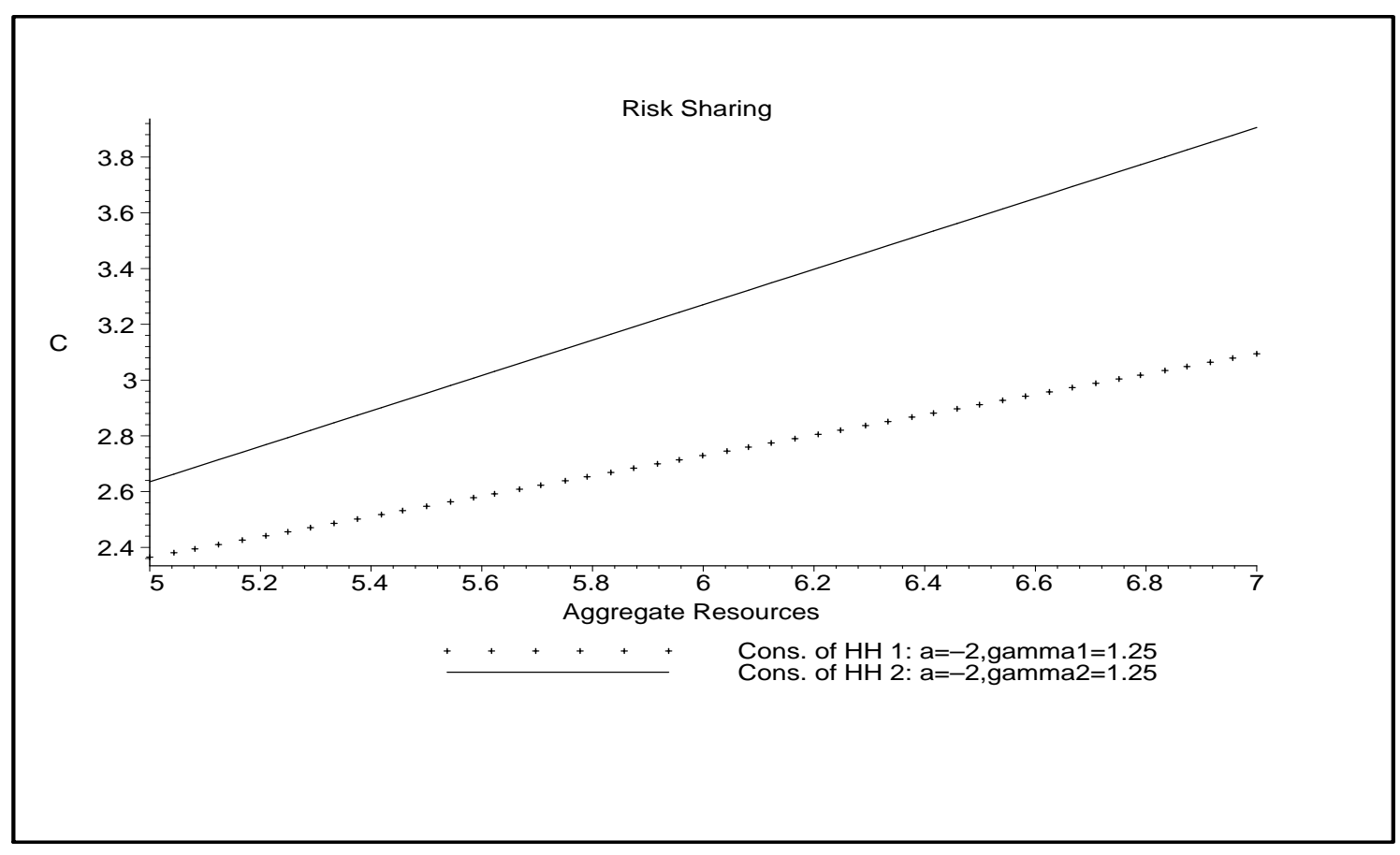


Figure 5: Expenditures as a Function of Aggregate Resources.
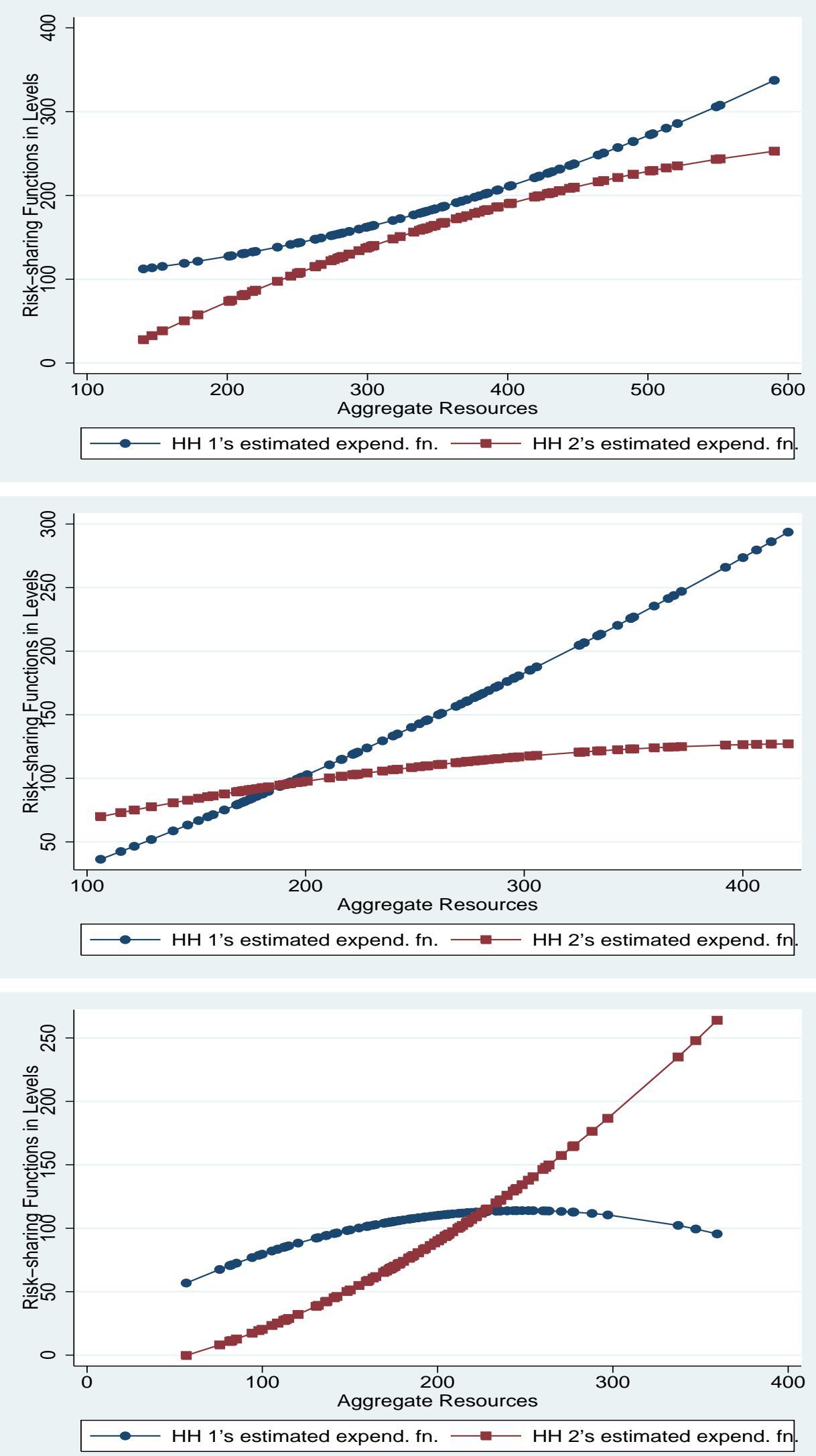
Figure 6: Decreasing Expenditures as a Function of Aggregate Resources.

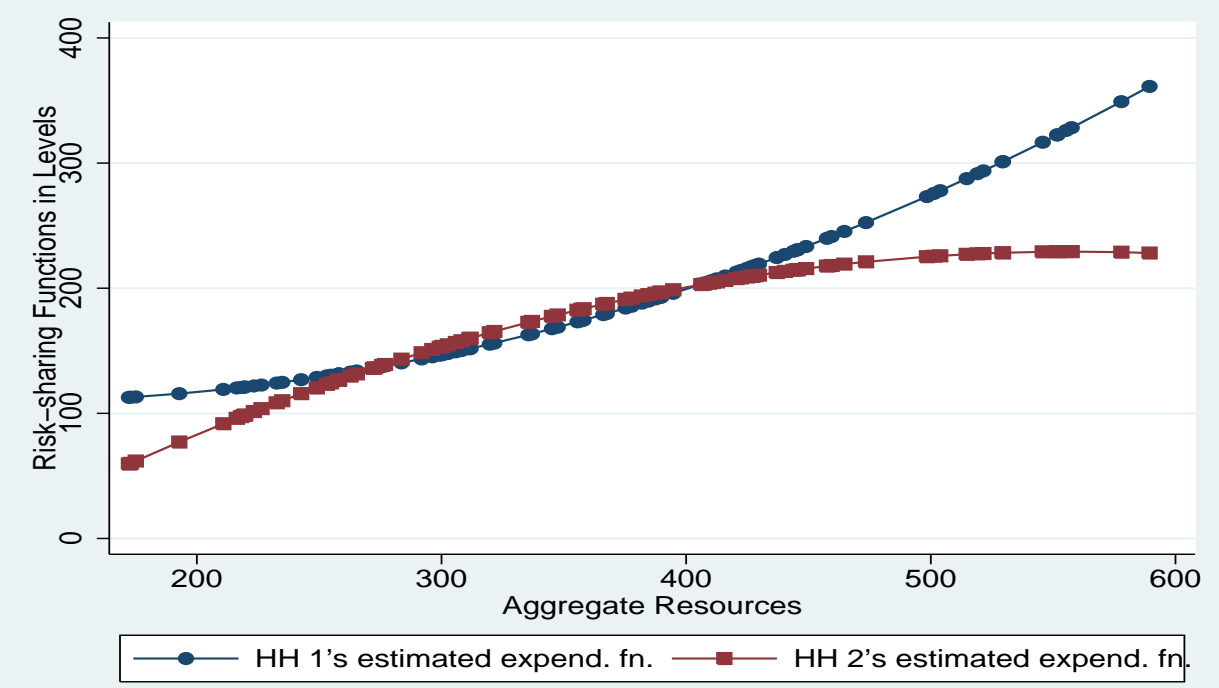

